\title{
TREATMENT OF PANCREATIC CANCER CELLS IN VITRO USING ULTRASOUND, MICROBUBBLES, AND GEMCITABINE
}

\author{
by \\ Julia Christine Mariglia \\ BSc (Hons), Ryerson University, 2014 \\ A thesis presented to Ryerson University \\ in partial fulfillment of the \\ requirements for the degree of \\ Master of Science \\ in the Program of \\ Biomedical Physics
}

Toronto, Ontario, Canada, 2016

CJulia Christine Mariglia 2016 


\section{AUTHOR'S DECLARATION}

I hereby declare that I am the sole author of this thesis. This is a true copy of the thesis, including any required final revisions, as accepted by my examiners.

I authorize Ryerson University to lend this thesis to other institutions or individuals for the purpose of scholarly research.

I further authorize Ryerson University to reproduce this thesis by photocopying or by other means, in total or in part, at the request of other institutions or individuals for the purpose of scholarly research.

I understand that my thesis may be made electronically available to the public.

Julia Christine Mariglia 


\title{
ABSTRACT
}

\section{TREATMENT OF PANCREATIC CANCER CELLS IN VITRO USING ULTRASOUND, MICROBUBBLES, AND GEMCITABINE}

\author{
Julia Christine Mariglia \\ Master of Science, Biomedical Physics \\ Ryerson University, 2016
}

Ultrasonically stimulated microbubbles can enhance the localized delivery and cytotoxic effects of chemotherapy drugs to cells by transient permeabilization of cell membranes in a process called sonoporation. However, there is insufficient data investigating whether ultrasound and microbubbles (USMB) enhances the delivery and cytotoxicity of the nucleoside analog (NA) gemcitabine. To address this gap in the literature, cancer cells were sonicated using low frequency ultrasound in combination with Definity ${ }^{\circledR}$ microbubbles in the presence of NAs. Viability analyses show that gemcitabine in combination with USMB additively enhanced cell death, suggesting that these two therapies mediate cell death independent of one another. This was confirmed when USMB treatment did not enhance (nor impair) the retention of a radiolabeled NA molecule. Altogether, these data suggest that the laws of diffusion forcing small molecules across a barrier cannot solely describe the efficacy of sonoporation; there are obviously important biological factors specific to the molecule intended to be delivered to consider as well. 


\section{ACKNOWLEDGEMENTS}

First and foremost, I'd like to express my deepest gratitude to my supervisors Dr. Raffi Karshafian and Dr. Imogen Coe. Dr. Karshafian has been a mentor to me for four years, and I thank him for the guidance and patience he's had with me over the years as I took the time to develop as a scientist. Dr. Coe has taught me how to speak confidently about not only my research, but also myself. This project would not have been possible without the guidance and scientific contributions from both of my supervisors.

A special thanks to my committee members: Dr. Costin Antonescu and Dr. Michael Kolios. I am truly fortunate to have had two great scientific minds challenging and inspiring me for the duration of this project.

I'd also like to acknowledge Drs. Natàlia Grañé and Zlatina Tarmakova. These are two beautiful women who have been more than just co-workers. I thank them both for their scientific contribution to this project and also for their friendship. They have both gotten me through my best and worst times.

To all my Coe and Karshafian lab mates, thank you for the laughs and support. Especially to Shadab Momin for his development of the PCD system in our lab. This project would not be complete without him.

Chris Spring - there are not enough words to express my gratitude for his flow cytometry, cell sorting, (and I guess comedic) expertise.

Thanks to Arthur Worthington, who has not only been a pleasure to work with but has been very helpful in designing parts of the ultrasound setup.

And to all my family, friends, and Biomedical Physics buddies - thank you for your ongoing love, support, and encouragement that made working on this project much more enjoyable. 


\section{TABLE OF CONTENTS}

AUTHOR'S DECLARATION ................................................................................ ii

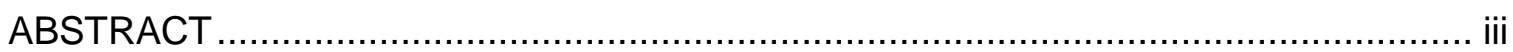

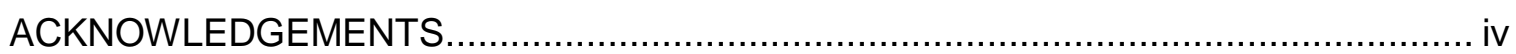

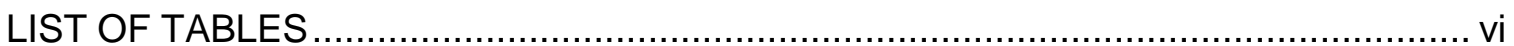

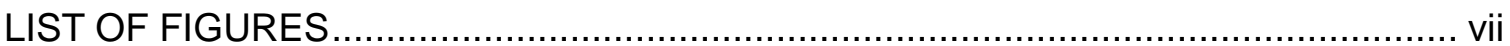

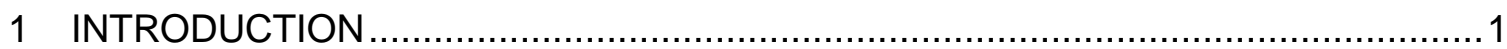

1.1 Rationale guiding this research............................................................................. 1

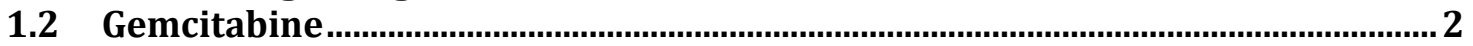

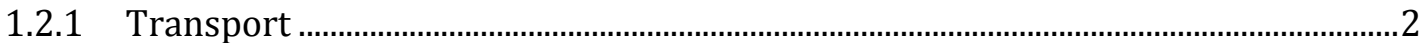

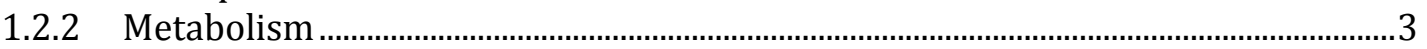

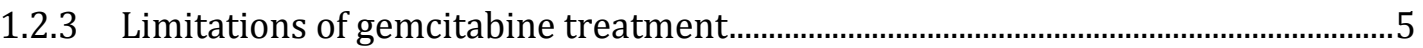

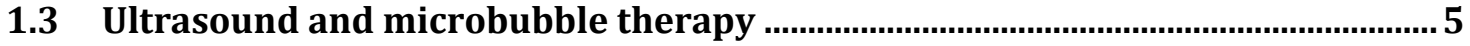

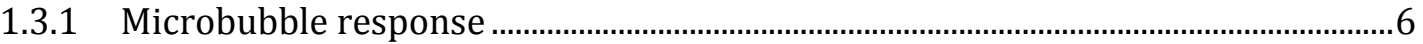

1.4 Ultrasound therapy applications in medicine

1.5 Hypothesis and Specific Objectives................................................................. 11

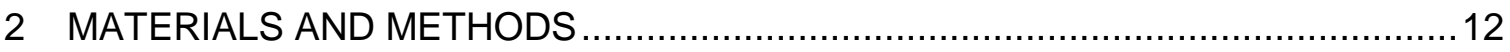

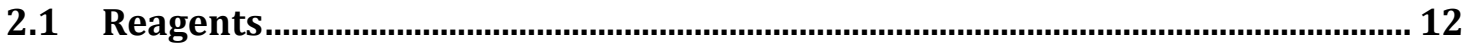

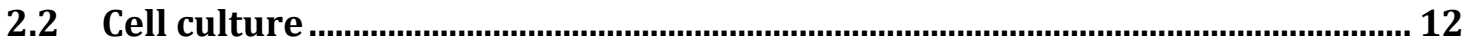

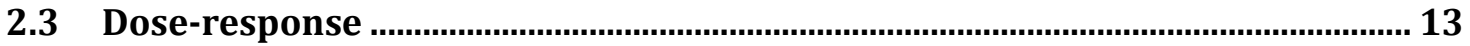

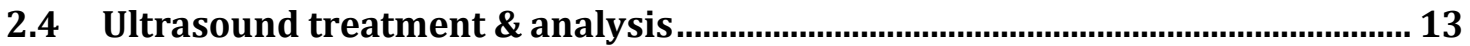

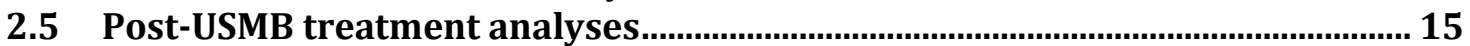

2.5.1 Cell viability with the MTT assay................................................................................. 15

2.5.2 Nucleoside analog uptake with cell sorting …........................................................ 15

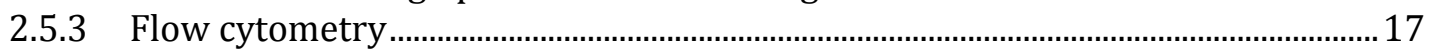

2.6 Passive Cavitation Detection ........................................................................... 18

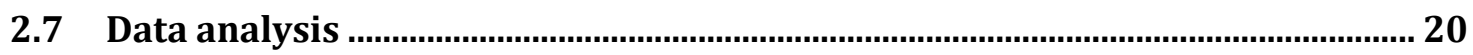

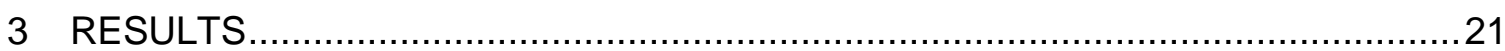

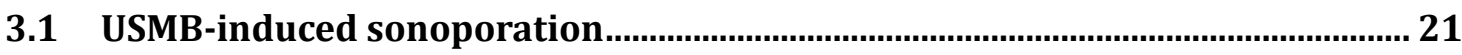

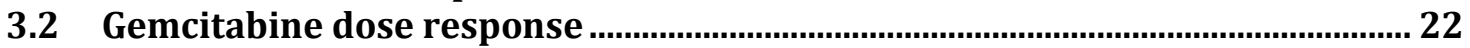

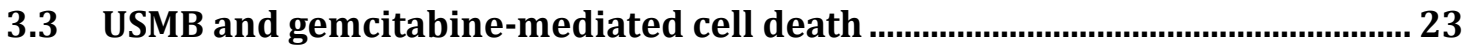

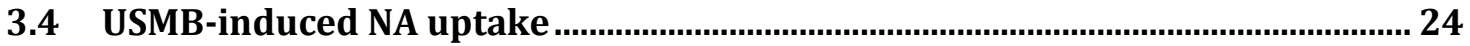

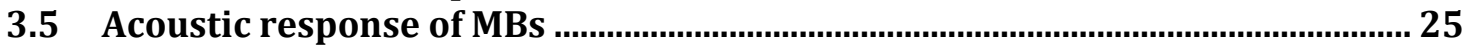

3.5.1 Acoustic emission correlations with USMB-induced bioeffects ...............................28

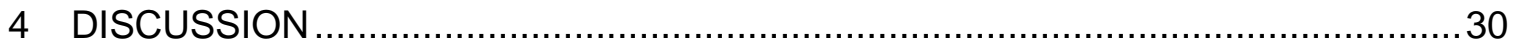

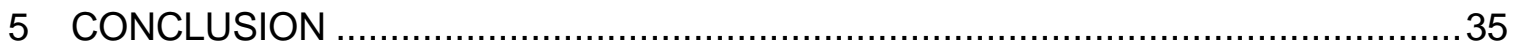

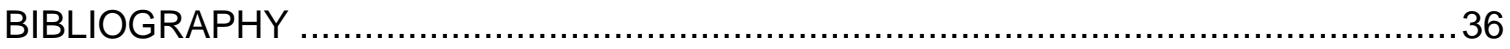




\section{LIST OF TABLES}

Table 3-1: $\mathrm{R}^{2}$ values for correlations made between MB behaviour and USMB-induced

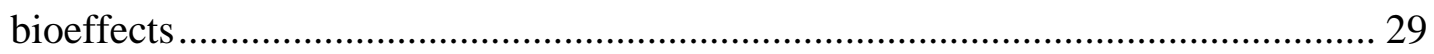




\section{LIST OF FIGURES}

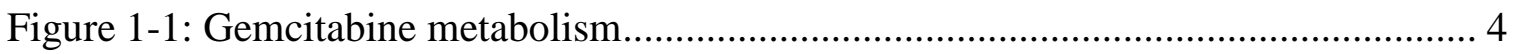

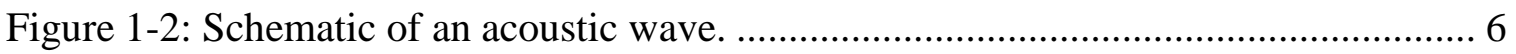

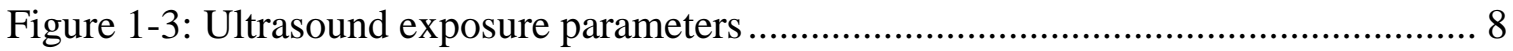

Figure 2-1: Experimental setup for USMB treatment........................................... 14

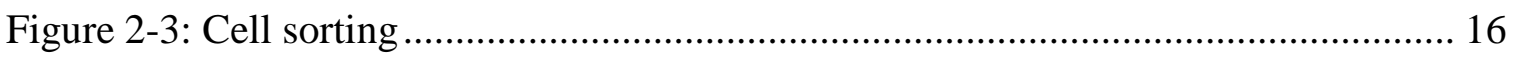

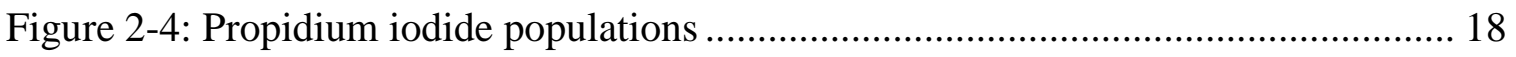

Figure 2-5: Experimental setup for PCD analyses................................................. 19

Figure 3-1: USMB-induced sonoporation with FITC-dextran ................................... 22

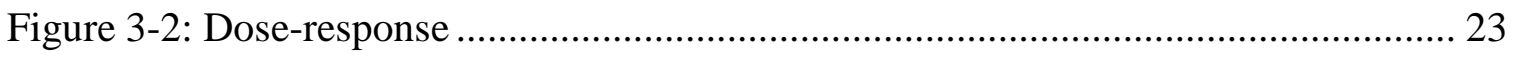

Figure 3-3: Cell viability of the combination therapy ............................................... 24

Figure 3-4: Intracellular 2-Chloroadenosine uptake ............................................... 25

Figure 3-5: Representative frequency spectra for each PNP …............................... 27

Figure 3-6: Wideband acoustic emission as a function of increasing PNP ................... 28

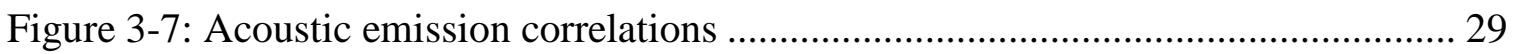




\section{INTRODUCTION}

\subsection{Rationale guiding this research}

In the last twenty years, scientists have been reporting on the use of ultrasound as a tool to enhance the delivery of drugs ${ }^{1-3}$, gene products $^{4-8}$, and fluorescent markers ${ }^{5,9}$ into cells by transient permeabilization of cell membranes. This phenomenon is defined as sonoporation, and its efficiency is enhanced when ultrasound is used in the presence of microbubbles (MBs), small gas filled bodies that oscillate in response to ultrasound. The ultrasound parameters partly dictate the acoustic response of MBs, which in turn dictates the bioeffects induced on cells that enhance molecular uptake ${ }^{10}$.

The potential for this approach to enhance drug delivery is the focus of this research. A major limitation with current drug delivery methods is maximizing the delivery of drug to the area of interest (such as a tumour) while sparing healthy cells from exposure to drug ${ }^{9,11}$. Studies have shown that ultrasound and microbubble (USMB) therapy can be focused with millimeter precision ${ }^{11,12}$ to enhance the localized uptake of chemotherapy drugs and representative fluorescent markers in vitro ${ }^{1,9,13,14}$ and in vivo ${ }^{15-}$ 17, paving the way for clinical studies employing this therapy to enhance drug delivery $^{18,19}$. Despite these studies, there is insufficient data on the effect of USMB therapy on enhancing the delivery of a major class anti-cancer, anti-viral, and antiparasitic drugs: the nucleoside analogs (NA) and in particular, the widely used anticancer drug, gemcitabine. 


\subsection{Gemcitabine}

\subsubsection{Transport}

Gemcitabine (2',2'-difluorodeoxycytidine - $\mathrm{dFdC}$ ) is a pyrimidine (deoxycytidine) nucleoside analog chemotherapy drug used in the treatment of a number of solid cancers. Nucleosides (and analogs) are hydrophilic, thus their diffusion across cell membranes is negligible, if at all possible ${ }^{20-22}$. To assist in salvaging these DNA and RNA precursors, transport systems evolved to facilitate nucleoside entry and efflux ${ }^{21}$. There exist two human Nucleoside Transporter (NT) families: the human Concentrative Nucleoside Transporters (hCNT), encoded by the SLC28 gene family, and the human Equilibrative Nucleoside Transporters (hENT), which are encoded by the SLC29 gene family. The pharmacological significance of these NTs is that nucleoside analog drugs such as gemcitabine use these transport systems to facilitate their entry to cells.

The SLC28 family of proteins transports its substrates unidirectionally into cells, actively driven by an inwardly directed sodium gradient (or in some cases a proton gradient $)^{22}$. The SLC28 family comprises three different genes that encode three different CNT proteins (CNT1-3), each of them differing in the specificity and affinity with which they transport their substrates. CNT1 predominantly transports pyrimidine nucleosides (including gemcitabine) and adenosine $e^{23,24}$. CNT2 specifically transports purines and uridine and CNT3 has a broad selectivity for both pyrimidines and purines ${ }^{23}$.

The SLC29 family is comprised of four different genes in humans that encode four different ENT proteins (ENT1-4). The ENT family facilitates the transport of its substrates bidirectionally across the cell membrane, along their concentration gradient. hENT1 and hENT2 are most ubiquitously expressed in human tissues and have a broad 
selectivity for both pyrimidine and purine nucleoside transport, including gemcitabine ${ }^{25}$. hENT2 differs from hENT1 in that it is also selective for purine and pyrimidine nucleobase transport ${ }^{26}$. hENT3 also transports a broad range of both purines and pyrimidines, but does so intracellularly with little apparent significance on nucleoside entry across the plasma membrane ${ }^{26}$. hENT4 predominantly functions in adenosine transport and as a monoamine transporter ${ }^{27}$. Both hENT3 and hENT4 are the most recently identified and are not fully characterized.

Gemcitabine is transported into cells by hENT1, hCNT1, hCNT3, and, with lower affinity, by hENT2. The presence of these NTs at the cell membrane is critical for the clinical efficacy of gemcitabine ${ }^{22}$. In fact, deficiencies in the NTs required to transport gemcitabine have been correlated with gemcitabine resistance, which has been shown in vitro and in clinically relevant situations ${ }^{4,28-33}$. For example, higher hENT1 protein levels in pancreatic cancer patients treated with gemcitabine correlated with longer median survival than patients deficient in hENT1 ${ }^{32}$. Similar conclusions were drawn from Santini et al.'s work where they showed that hENT1 levels were predictive biomarkers of the efficacy of gemcitabine treatment in patients with biliary tract cancer ${ }^{33}$. In addition, Paproski et al. confirmed that cells lacking functional NTs are resistant to gemcitabine treatment, but can become sensitized to gemcitabine when cells were transfected with the hCNT3 gene using sonoporation methods ${ }^{4}$.

\subsubsection{Metabolism}

Once gemcitabine is transported into a cell, it is phosphorylated by deoxycytidine kinase (dCK) to gemcitabine monophosphate and subsequently phosphorylated by other kinases to gemcitabine di- and tri-phosphate ${ }^{20}$. The di- and tri-phosphate forms of the 
drug ( $\mathrm{dFdCDP}$ and $\mathrm{dFdCTP}$, respectively) are considered to be the "active" metabolites, as they are involved in terminating DNA replication. dFdCTP competes with deoxycytidine-5'-triphosphate (dCTP) to be incorporated into a growing strand of $\mathrm{DNA}^{34}$, after which another nucleotide is added before strand termination occurs ${ }^{35}$. dFdCDP inhibits a multisubunit enzyme, ribonucleotide reductase (RNR), which converts ribonucleotide diphosphates (NDPs) into deoxyribonucleotide diphosphates (dNDPs) to be used during DNA replication and repair processes. As a result, $\mathrm{dFdCDP}$ inhibits the de novo synthesis of DNA substrates. In this way, the likelihood of dFdCTP incorporation into a growing strand of DNA is increased by decreasing its competition with other deoxyribonucleotides; this is referred to as a self-potentiated mechanism ${ }^{34}$. A summary of gemcitabine metabolism is depicted in Figure 1-1.

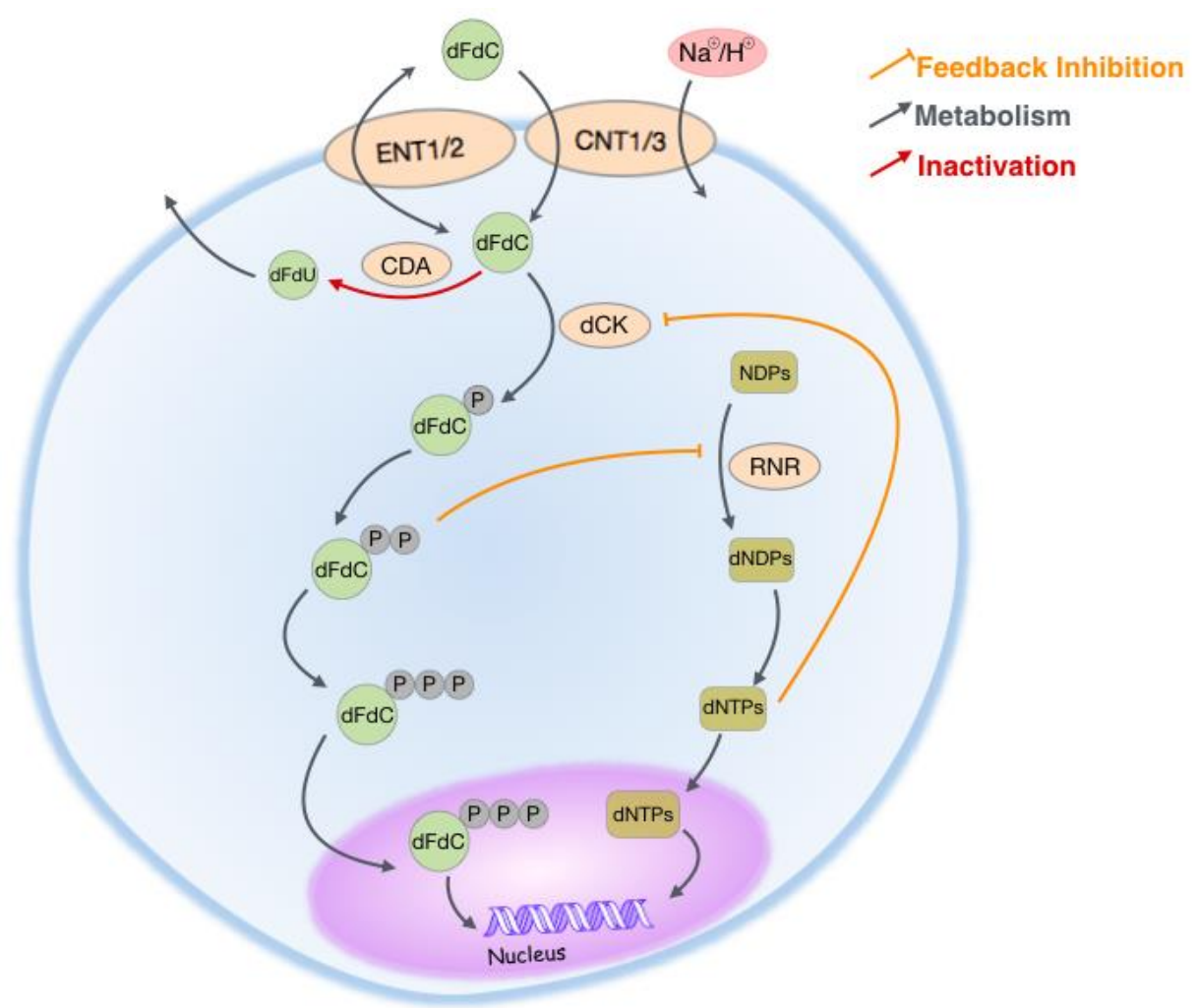

Figure 1-1: Gemcitabine metabolism (Redrawn from PharmGKB ${ }^{36}$ ). 


\subsubsection{Limitations of gemcitabine treatment}

As seen in Figure 1-1, gemcitabine is inevitably inactivated by the enzyme cytidine deaminase (CDA), which deaminates $\mathrm{dFdC}$ to 2',2'-difluorodeoxyuridine $(\mathrm{dFdU})$, a less toxic form of the drug ${ }^{37}$. There are also high levels of CDA in the blood plasma, which rapidly decreases the availability of active gemcitabine in the circulation that is required to induce cytotoxicity (half-life $\sim 8-20$ minutes) ${ }^{37-39}$. Secondly, systemic administration of gemcitabine causes all rapidly dividing cells in the body to be susceptible to the harmful effects of the drug, not just the cancer cells ${ }^{40}$. In addition, gemcitabine cytotoxicity is highly dependent on effective transport across the PM by NTs, thus the cytotoxic effects of this drug are limited by the presence of these functional transporters to permit gemcitabine entry into cells. Therefore, regulation and physiology of NTs may impact drug efficacy. With these limitations in mind, it is clear we need a more effective delivery method to reliably enhance the localized uptake of gemcitabine to the region of interest, thereby decreasing the circulation time within the body, and minimizing exposure of drug to healthy cells. Ultrasound and microbubble therapy can potentially be used as a treatment modality to overcome some of these current limitations of gemcitabine chemotherapy treatment.

\subsection{Ultrasound and microbubble therapy}

Ultrasound is a non-invasive modality that uses high frequency sound waves $(0.5$ $12 \mathrm{MHz})$ for diagnostic and therapeutic applications ${ }^{41}$. These sound waves are longitudinal waves, meaning that the particles within the medium that the wave is travelling in are displaced parallel to the direction of the travelling wave (Figure 1-2a). It is the local displacements of these particles within the media to and from their 
equilibrium position that allow the wave to propagate forward ${ }^{41}$. High particle density denotes regions of high pressure (or compression) and low particle density denotes low pressure (or rarefaction) regions.

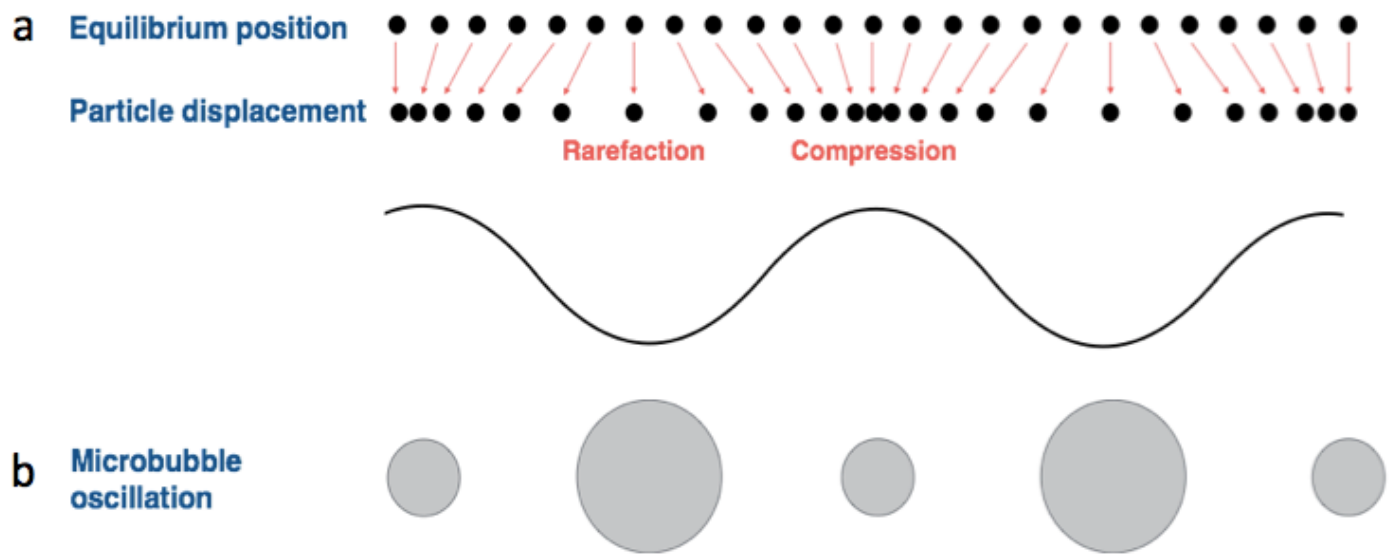

Figure 1-2: Schematic of an acoustic wave. a) The pressure gradient from an acoustic wave displaces particles in the medium it is propagating in from their equilibrium position into compressional and rarefactional regions. b) Microbubbles contract at the compressional phases of the ultrasound wave and expand at the rarefactional phases. Image redrawn from What is Ultrasound ${ }^{41}$.

Microbubbles are small (1-10 $\mu \mathrm{m}$ diameter size range) gas-filled bodies encapsulated within a shell composed of either lipid, polymer, or protein ${ }^{5,42}$. They were originally approved by the Food and Drug Administration (FDA) and Health Canada for their use as ultrasound contrast agents to enhance the scattering of ultrasound from blood $^{43}$ but ongoing research is investigating their role as cavitation nuclei for therapeutic applications as well.

\subsubsection{Microbubble response}

Microbubbles oscillate in response to the pressure gradient generated by ultrasound, expanding at the rarefactional phases of the wave and contracting at the 
compressional phases (Figure 1-2b). The extent to which bubbles expand and contract depends on the microbubble size, shell composition, compressibility, and the ultrasound parameters used to sonicate them.

Generally, at lower acoustic pressures, microbubble oscillation is defined as stable (or non-inertial) cavitation and the integrity of the bubble is preserved. A bubble can stably oscillate in a linear or non-linear manner. Stable linear oscillation refers to a symmetrical change in $\mathrm{MB}$ radius during expansion and contraction ${ }^{44}$. Stable non-linear oscillation occurs at slightly higher pressures than those used to induce stable linear oscillation and refers to when the $\mathrm{MB}$ expands at the rarefactional phases of the ultrasound wave, as it did in linear oscillation, but cannot contract without limit because the volume of gas within the microbubble limits further contraction ${ }^{42}$. Both stable linear and non-linear oscillations generate local flows in the surrounding fluid (called microstreaming), which can induce shear stress on cells, pushing and pulling effects, and translational effects, all of which can destroy the integrity of cellular plasma membranes and contribute to sonoporation ${ }^{5}$.

When the ultrasound pressure is further increased, microbubble behaviour is defined as inertial cavitation (IC). During oscillation, the rarefactional phase of the wave causes such a large expansion of the bubble that the inertia of the surrounding fluid causes it to collapse and/or fragment into many smaller bubbles ${ }^{5,42}$. This violent collapse of inertially cavitating bubbles can lead to the formation of shock waves and liquid jets $^{5,42}$.

Oscillating MBs emit unique acoustic signals that are associated with its acoustic response $^{45}$. Stable linear MB oscillations produce an acoustic signal typically at the same 
frequency as the transmitting transducer frequency (i.e. the fundamental frequency) ${ }^{46}$. Harmonic (as well as sub- and ultra-harmonic) signals are indicative of nonlinear MB behaviour $^{44,47-49}$. In addition, MBs undergoing inertial cavitation emit broadband acoustic signals ${ }^{50}$. These various acoustically emitted signals can be passively recorded with a transducer and can be used to characterize MB behaviour in response to different ultrasound parameters.

MB behaviour (and thus USMB-induced bioeffects) is governed by parameters other than just acoustic pressure, including transducer centre-frequency, pulse repetition frequency, pulse duration, and sonication time ${ }^{9}$. These terms are illustrated in Figure 1-3 below.

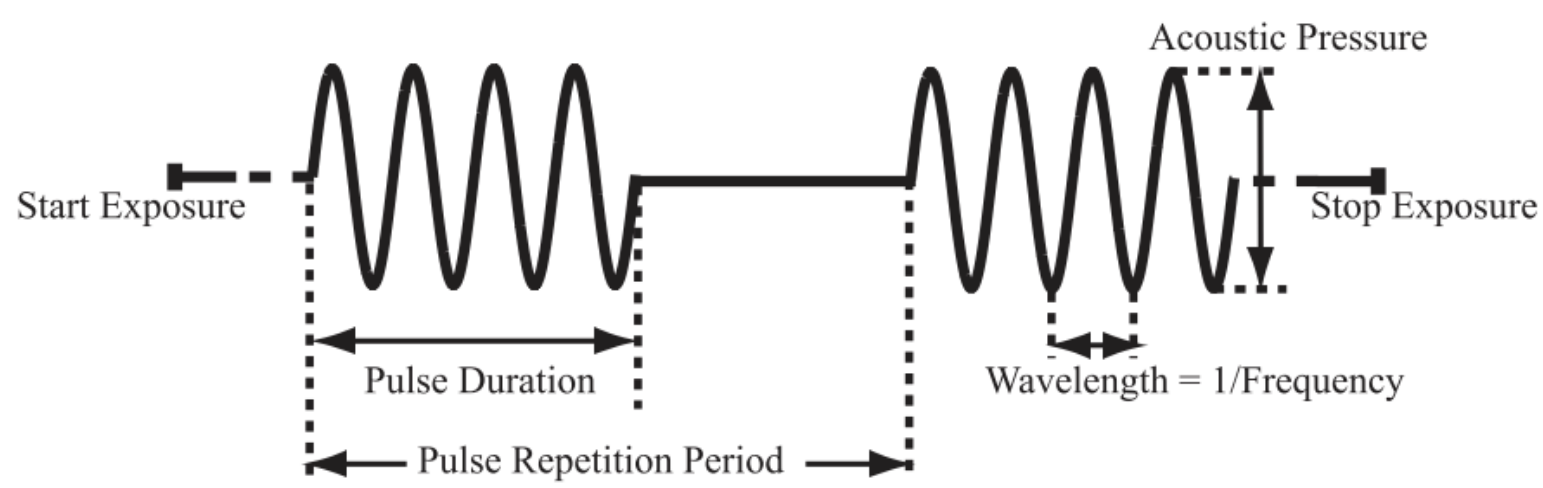

Figure 1-3: Ultrasound exposure parameters ${ }^{47}$.

\subsection{Ultrasound therapy applications in medicine}

Some current FDA approved modalities employing ultrasound therapy include i) physiotherapy where ultrasound is used to accelerate healing of soft tissue injuries, ii) high intensity focused ultrasound (HIFU) in tissue ablation, and iii) lithotripsy, which relies on the effects of mechanical stress induced and cavitation mechanisms ${ }^{51}$. Although 
both ultrasound and microbubbles are clinically approved tools for imaging, their combination as a therapeutic modality is not. As discussed, the mechanism of this therapy depends on the mechanical effects induced by microbubble oscillations to enhance the delivery of drugs and biological molecules. However, the ultrasound conditions that are required to induce a desirable microbubble response and biological effect are not well known, and further research is required.

Nonetheless, the literature contains a plethora of data in support of the potential for this therapy to be used as a reliable tool to enhance drug delivery for cancer treatment. Numerous in vitro studies have set the foundation by showing in a controlled setting that USMB is capable of enhancing intracellular chemotherapy delivery. For instance, Lentacker et al. have demonstrated that targeted MBs can synergistically enhance the intracellular accumulation of the chemotherapy drug Doxorubicin (DOX), which correspondingly resulted in an enhancement in cell death ${ }^{1}$. A seemingly straightforward but fundamental study by Escoffre et al. shared similar conclusions where ultrasonically stimulated MBs can enhance the delivery of free DOX to cell suspensions as well, causing DOX to passively diffuse across the transient pores formed in the cell plasma membrane ${ }^{52}$. The resulting success of these controlled studies was the motivation for researchers to be able to move forward and successfully facilitate chemotherapy delivery in vivo to (i) show significant reductions in tumour volume $e^{16,53}$ and to (ii) reversibly permeabilize the blood-brain barrier ${ }^{54,55}$. Because of these advancements over the years in the field of USMB treatment, scientists are now in the clinical stages of utilizing this combination therapy on patients ${ }^{2,18}$. 
To the best of our knowledge, there are only two other studies that have investigated the cytotoxic effects of USMB in combination with gemcitabine ${ }^{16,18}$. In one in vivo study, Kotopoulis et al. have shown a significant reduction in pancreatic tumour volume and a slight (but insignificant) increase in overall survival in mice treated with the combination therapy ${ }^{16}$. A clinical study employing these two treatment modalities also showed that the combined therapy modestly enhanced the number of treatment cycles patients were able to receive (indicative of patient health) which extended overall patient survival by one month in comparison to a historical control group of 80 patients whom received treatment with gemcitabine alone. While both of these studies demonstrated the potential for sonoporation to be a feasible strategy to improve the effectiveness of chemotherapy treatment with gemcitabine, neither actually verified that the clinical benefits were attributed to an enhancement in gemcitabine delivery. Thus, the purpose of this study was to determine if USMB therapy actually enhances the uptake (and cytotoxicity) of gemcitabine to cancer cells. 


\subsection{Hypothesis and Specific Objectives}

The hypothesis of this research is that ultrasound and microbubble exposure will enhance the cytotoxic effects of nucleoside analogs in cancer cells in vitro.

The specific objectives of this study were:

1. To determine the effect of USMB therapy in combination with gemcitabine on cell viability.

2. To determine if USMB therapy enhances the uptake of NAs into cells.

3. To determine if the efficacy of the combined USMB and NA therapy is dependent on the behaviour of oscillating MBs. 


\section{MATERIALS AND METHODS}

\subsection{Reagents}

Gemcitabine hydrochloride was purchased from Tocris Bioscience (Bristol, UK) and freshly diluted in serum-containing growth media before each use. Fluorescein isothiocyanate (FITC) dextran, (10 kDa, Cat. No. D1820), propidium iodide (PI, Cat. No. P3566), Fetal Bovine Serum (FBS), and 0.05\% trypsin-EDTA were purchased from Life Technologies Inc. (Burlington, ON). Roswell Park Memorial Institute (RPMI-1640) growth media and Phosphate Buffered Saline (PBS) were purchased from Princess Margaret Hospital Media Services (Toronto, ON). Thiazolyl Blue Tetrazolium Bromide (MTT) powder (Cat. No. M2128-1G) and 2-Chloroadenosine (Cat. No. C5134) were purchased from Sigma-Aldrich (St. Louis, Mo). Radiolabeled 2-Chloroadenosine (MT697) was purchased from Moravek Biochemicals (Brea, CA). Dimelthyl sulfoxide (DMSO) was purchased from Bio Basic (Cat. No. D0231). HEPES buffer was purchased from Wisent Inc. (Saint-Jean-Baptiste QC). Plasmocin was purchased from Cedarlane Labs (Burlington, ON).

\subsection{Cell culture}

The BxPC3 pancreatic adenocarcinoma cell line was chosen as it is a clinically relevant cell model. This cell line was also responsive to gemcitabine treatment, which ensured that these cells had the enzymatic capacity to metabolize gemcitabine. Cells were cultured in RPMI-1640 growth media supplemented with 10\% (v/v) FBS, incubated at $37^{\circ} \mathrm{C}$ with $5 \% \mathrm{CO}_{2}$ and passaged at $80-90 \%$ confluence using $0.05 \%$ trypsin-EDTA. This cell line was demonstrated to be mycoplasma-free and the cells were grown in media containing $0.1 \%$ (v/v) Plasmocin; Plasmocin was removed 24 hours prior to when 
cells were used for experiments. Cells were trypsinized for 5 minutes, centrifuged (1000 rpm for 4 minutes) to remove excess trypsin, and resuspended in fresh serum-containing media before being used for experiments.

\subsection{Dose-response}

For dose-response analyses, BxPC3 cells $\left(1.5 \times 10^{6}\right.$ cells $/ \mathrm{mL}$ in a total volume of $0.6 \mathrm{~mL}$ ) were exposed to increasing concentrations of gemcitabine for 2 hours in a humidified incubator set at $37^{\circ} \mathrm{C}$ and $5 \% \mathrm{CO}_{2}$. Following incubation, samples were centrifuged and washed twice with cold PBS to remove excess gemcitabine (1000 rpm for 4 minutes), diluted appropriately in serum-containing media, and plated in replicates into a 96 -well plate (8000 cells per well) in eight replicates. After 48 hours, the MTT assay was performed to quantify cell viability. The interpolated IC50 dose was used for further experiments.

\subsection{Ultrasound treatment \& analysis}

The ultrasound setup consisted of a $0.5 \mathrm{MHz}$ single-element focused transducer (IL0.508HP, Valpey Fisher Inc., Hopkinton, MA) connected to a power amplifier (T\&C Power Conversion Inc., Rochester, NY) and arbitrary waveform generator (AWG; Model WW5062, Tabor Electronics Ltd., Irvine, CA), controlled by ArbConnection computer software (Tabor Electronics Ltd., Irvine, CA). The transducer was submerged in deionized (DI) and degassed water and positioned at the focus of the sample chamber's acoustic window. Ultrasound treatment was performed at three peak negative pressures (PNP: 220, 360, and $530 \mathrm{kPa}$ ) with $1.7 \%$ (v/v) Definity ${ }^{\circledR}$ microbubbles, yielding a 
MB:cell ratio of 100 (Lantheus Medical Imaging, N. Billerica, MA). The microbubbles were activated using a VialMix ${ }^{\circledR}$ for 45 seconds and diluted in PBS.

Cells $\left(1.5 \times 10^{6}\right.$ cells $/ \mathrm{mL}$ in a total volume of $\left.0.6 \mathrm{~mL}\right)$ were treated with USMB in the presence of $1 \mu \mathrm{M}$ gemcitabine, 2-Chloroadenosine (plus $12.1 \mathrm{Ci} / \mathrm{mmol}\left[{ }^{3} \mathrm{H}\right]-2$ Chloroadenosine), or $10 \mathrm{kDa}$ FITC dextran. The 2-Chloroadenosine and FITC-dextran concentrations correspond with the IC50 of gemcitabine used in chemotherapy experiments. Cells were sonicated for 1 minute with a pulsed 16-cycle wave and a pulse repetition period (PRP) of $1 \mathrm{~ms}$. The duty cycle of the pulse was 3.2\%. A schematic of the ultrasound setup is depicted in Figure 2-1.

Immediately following USMB treatment, samples were incubated for 2 hours, after which they were centrifuged and washed twice (1000 rpm for 4 minutes) with cold PBS (for cell viability and flow cytometry measurements) or sodium free buffer (for scintillation counting) before the appropriate assay was performed.

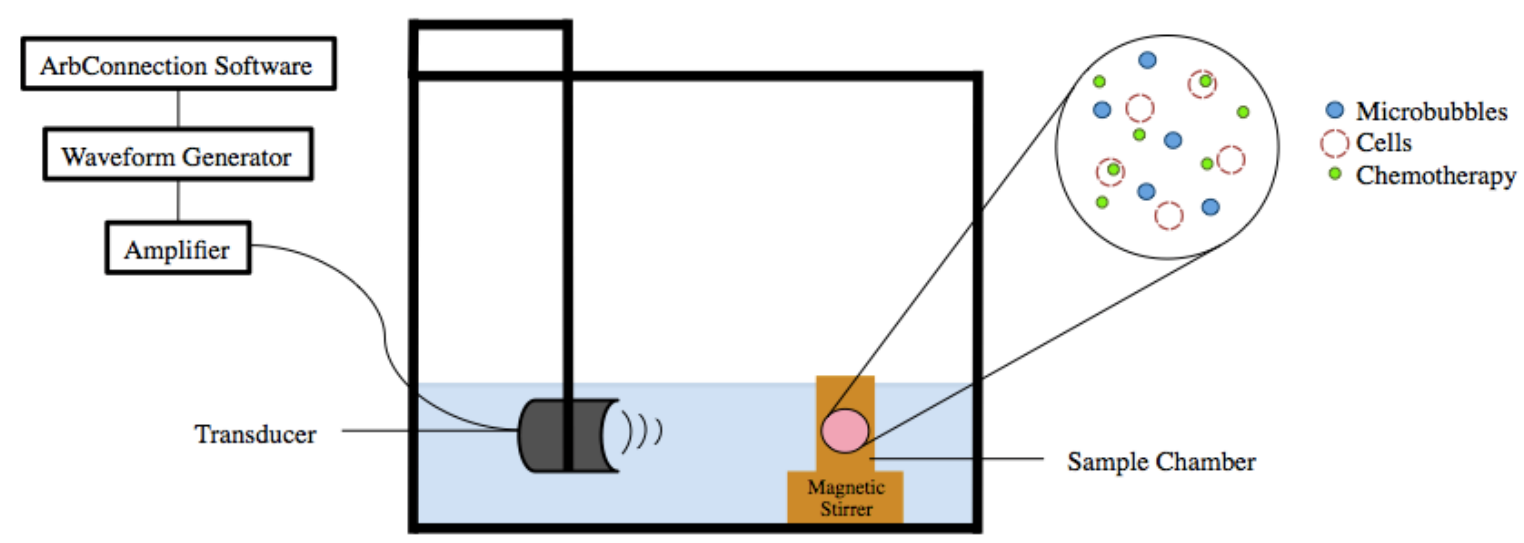

Figure 2-1: Experimental setup for USMB treatment. A $0.5 \mathrm{MHz}$ focused transducer was submerged in water and focused at the sample chamber's acoustic window. A magnetic stirrer thoroughly homogenized the cell samples during treatment. 


\subsection{Post-USMB treatment analyses}

\subsubsection{Cell viability with the MTT assay}

The MTT (3-[4,5-dimethylthiazol-2-yl]-2,5-diphenyltetrazolium bromide) assay is a colorimetric cell viability assay. Mitochondrial reductases reduce a yellow tetrazolium dye into an insoluble purple formazan. It is presumed that the quantity of formazan (an indicator for cell metabolic activity) is directly proportional to the number of viable cells.

Following the 2-hour incubation and centrifugation, cell samples that were treated with $1 \mu \mathrm{M}$ of gemcitabine were seeded in a 96-well plate (8000 cells per well) in eight replicates for 48 hours. After 48 hours, the media in each well was aspirated and $100 \mu \mathrm{L}$ of MTT solution $(0.75 \mathrm{mg} / \mathrm{mL})$ was added to each well. After 1-hour of incubation, the MTT solution was aspirated and the intracellular purple formazan was solubilized with $100 \mu \mathrm{L}$ DMSO. The plate was gently shaken until the purple dye in each well was homogenous in colour (10-15 minutes), at which point the amount of formazan was quantified with an iMark Microplate Absorbance Reader (Bio-rad, Hercules, CA) operating at $540 \mathrm{~nm}$.

\subsubsection{Nucleoside analog uptake with cell sorting}

Following the 2-hour incubation and centrifugation, cell samples that were treated with $1 \mu \mathrm{M}$ of 2-Chloroadenosine (plus $12.1 \mathrm{Ci} / \mathrm{mmol}$ of $\left[{ }^{3} \mathrm{H}\right]$-2-Chloroadenosine) were centrifuged and washed twice with cold sodium free buffer $(20 \mathrm{mM}$ Tris- $\mathrm{HCl}, 3 \mathrm{mM}$ potassium diphosphate, $1 \mathrm{mM}$ magnesium chloride, $2 \mathrm{mM}$ calcium chloride, $5 \mathrm{mM}$ glucose, $130 \mathrm{mM} \mathrm{N-methyl} \mathrm{D-glucamine} \mathrm{(pH} \mathrm{7.4)).} \mathrm{After} \mathrm{the} \mathrm{second} \mathrm{centrifugation,} \mathrm{the}$ pellet was resuspended in $0.6 \mathrm{~mL}$ of PBS containing $20 \mathrm{mM}$ HEPES buffer and $5 \%$ FBS and kept on ice. A volume of $1.5 \mu \mathrm{L}$ of $1.0 \mathrm{mg} / \mathrm{mL}$ PI was added to each of these 
samples and thoroughly mixed to ensure a homogenous distribution of PI with the cells. PI is a red fluorescent molecule that is only permeable to cells with a compromised cell membrane (i.e. necrotic and late apoptotic cells). Cells were sorted according to PI accumulation to separate the cells that survived USMB treatment (PI negatively stained) from those that were irreversibly permeabilized (PI positively stained). Cell sorting was performed using a BD FACSAria III cell sorting system (BD Biosciences, San Jose, CA) where samples were excited with a $488 \mathrm{~nm}$ laser and passed through a 100- $\mu \mathrm{m}$ nozzle with a sheath pressure of 20 pounds per square inch. Cell droplets were generated at a rate of 30,000 per second and acquired at 1,000 events per second. While sorting, all other samples were kept on ice. Figure 2-2 describes the cell sorting procedure.
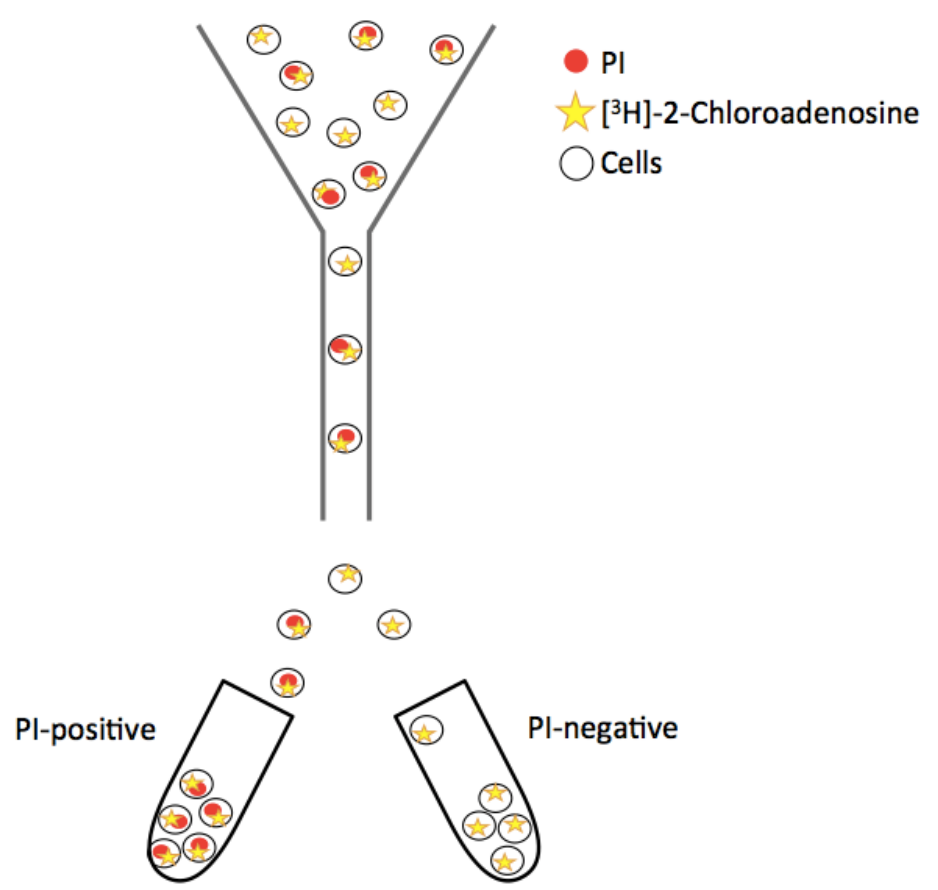

Figure 2-2: Cell sorting was performed with the BD FACS Aria III cell sorter where viable cells were sorted from necrotic cells according to PI fluorescence following USMB treatment. 
After cell sorting, samples were brought to the same final volume. One milliliter of each sample was lysed with $250 \mu \mathrm{L}$ of $2 \mathrm{M} \mathrm{NaOH}$ and stored overnight at $4^{\circ} \mathrm{C}$. The following day, $600 \mu \mathrm{L}$ of each sample was neutralized with $50 \mu \mathrm{L}$ of $8 \mathrm{~N} \mathrm{HCl}$ and transferred into scintillation vials (in duplicate). Five millilitres of ECOSCINT (Diamed Lab Services Inc., Mississauga, ON) scintillation solution was added to each vial. Radioactive accumulation due to transporter and USMB-mediated uptake was measured by standard scintillation counting with the Beckman LS6500 Multipurpose scintillation counter (Fullerton, CA). Total concentrations of radioactive 2-Chloroadenosine incorporated into cells were normalized to the total cell number within each sample obtained from cell sorting.

\subsubsection{Flow cytometry}

The LSRFortessa ${ }^{\mathrm{TM}}$ X-20 (BD Biosciences, San Jose, CA) was used to assess cell permeabilization and the intracellular concentration of a fluorescent molecule $(1 \mu \mathrm{M}$ of $10 \mathrm{kDa}$ FITC dextran) following USMB treatment. Following the 2-hour incubation and centrifugation, $1.5 \mu \mathrm{L}$ of $1.0 \mathrm{mg} / \mathrm{mL}$ PI was added to the cell samples and thoroughly mixed. Cells were excited with the $488 \mathrm{~nm}$ laser and FITC and PI emissions were detected with the 530/30 $\mathrm{nm}$ and 610/20 nm filters, respectively. Colour compensation was performed on unstained and single colour-stained cells. Propidium iodide positive and negative populations were sorted according to Figure 2-4 to emulate cell sorting in the same manner described previously. Percentage of FITC-permeabilized cells and mean fluorescence intensities (MFI) were calculated within these two populations. 


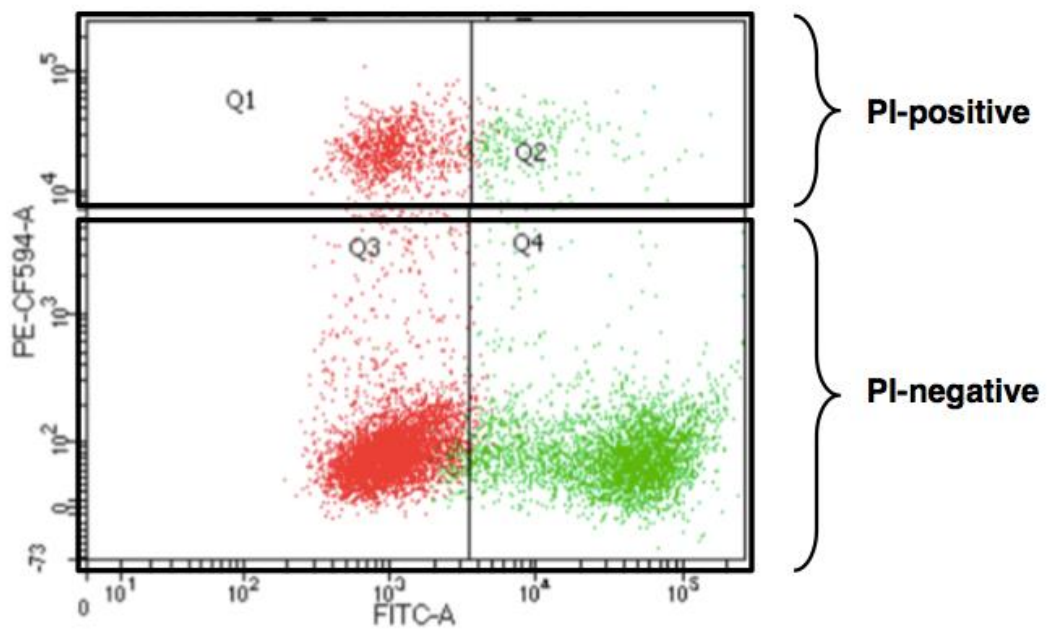

Figure 2-3: Propidium iodide populations were sorted as shown above for cells exposed to USMB in the presence of FITC-dextran. The two-parameter histogram above shows the logarithm of propidium iodide fluorescence (PE-CF594, y-axis) in relation to FITC fluorescence (x-axis).

\subsection{Passive Cavitation Detection}

A passive cavitation detection (PCD) system was used to measure the acoustic signals emitted from cavitating Definity ${ }^{\circledR}$ MBs during the various USMB treatments described previously. This technique was used to indicate if there was a correlation between MB activity and USMB-induced bioeffects. Note that the PCD experiments were conducted independently from other USMB experiments and in a different ultrasound setup.

A schematic of the PCD experimental setup is shown in Figure 2-4. The setup consisted of a $0.5 \mathrm{MHz}$ focused transmitting transducer and a focused $1 \mathrm{MHz}$ passive receiving transducer (IL0112HP, Valpey Fisher Inc., Hopkinton, MA). Both transducers were aligned at the focus of the sample chamber, 90-degrees from one another, and submerged in DI and degassed water. The sample chamber contained cells $\left(1.5 \times 10^{6}\right.$ cells $/ \mathrm{mL}$ in a total volume of $1.5 \mathrm{~mL})$ in the presence of $1.7 \%(\mathrm{v} / \mathrm{v})$ Definity ${ }^{\circledR}$ MBs. The 
equipment and treatment conditions for the transmitting $(0.5 \mathrm{MHz})$ transducer were the same as those described above. The receiving transducer was used as a passive cavitation detector to acquire the acoustic emissions from the MBs during USMB treatment. This 1 $\mathrm{MHz}$ transducer was connected to a $60 \mathrm{~dB}$ low noise amplifier (Model A150, Miteq, USA), a 3.5 MHz low-pass filter and the CompuScope MATLAB SDK (Mathworks Inc., Natick, MA) computer program.

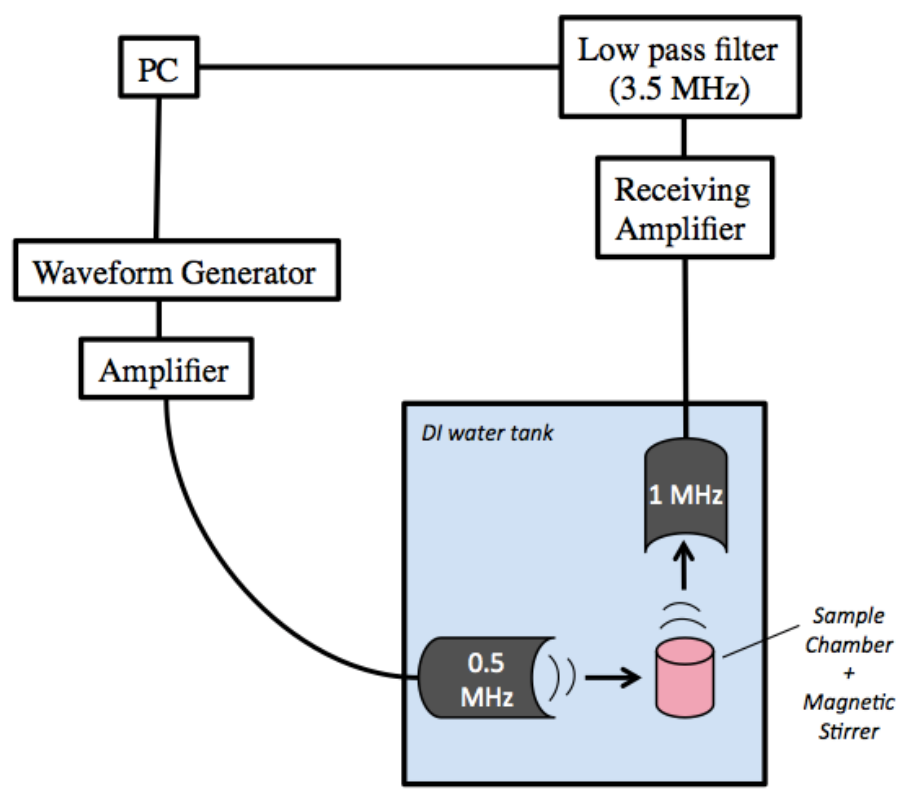

Figure 2-4: Experimental setup for PCD analyses. A $0.5 \mathrm{MHz}$ focused transducer acoustically stimulated the MBs within the cell sample chamber and a $1.0 \mathrm{MHz}$ transducer (oriented $90^{\circ}$ relative to the other) detected the MB acoustic emissions.

For each treated sample, 12,000 frequency spectra were generated from the corresponding time-domain (TD) signals using a fast Fourier transform (FFT). Twelvethousand frequency spectra were also obtained from three reference samples (one for each ultrasound PNP) which contained cells sonicated in the absence of MBs. Each reference signal's frequency-domain spectrum was subtracted from the corresponding 
sample's MB-treated frequency-domain spectrum. For each of the 12,000 final subtracted plots, the wideband area under the curve (AUC) was taken between 0.875 and 1.625 $\mathrm{MHz}$ and plotted as a function of the 60 -second total treatment time. The integral of this new AUC-versus-time plot (i.e. summation of these values over the 60 second treatment time) yielded a numerical value that represents $\mathrm{MB}$ acoustic emissions during sonication.

\subsection{Data analysis}

A one-way ANOVA was used to determine significance within PI or GEM populations. A two-way ANOVA followed by a Bonferroni's multiple comparisons posthoc test was used to determine statistical differences between PI or GEM populations, where P-values less than 0.05 were considered significant. R-squared values were calculated to assess correlations between the acoustic emission signals and USMBinduced bioeffects. 


\section{RESULTS}

The data produced in this study support the contention that USMB in combination with gemcitabine (GEM) resulted in an additive cytotoxic effect in BxPC3 pancreatic cancer cells. Although these ultrasound settings were sufficient to induce sonoporation of a fluorescent molecule (10 kDa FITC-dextran), USMB therapy did not enhance the intracellular concentration of the radiolabeled NA 2-Chloroadenosine. This suggested that USMB, at the settings used in this work, does not enhance nor impair the delivery and metabolism of NAs into cells.

\subsection{USMB-induced sonoporation}

We first verified that the ultrasound conditions were sufficient to induce sonoporation using $1 \mu \mathrm{M}$ of FITC-dextran. The percentage of FITC-positive cells increased with increasing ultrasound PNP in both PI-negative and PI-positive populations (Figure 3-1a, $P<0.0001$ and $P=0.0001$, respectively). There were always significantly more cells that were reversibly (PI-negative) permeabilized than irreversibly (PI-positive) permeabilized (Figure 3-1a, $* P<0.0001$ ). Not only did the number of permeabilized cells increase with PNP, but the average intracellular FITC concentration (MFI) also increased with increasing PNP for cells reversibly permeabilized (Figure 3-1b, $P<0.0001$ ). There was a negligible increase in the average intracellular FITC concentration for the PIpositively stained population $(P=0.1201)$. 

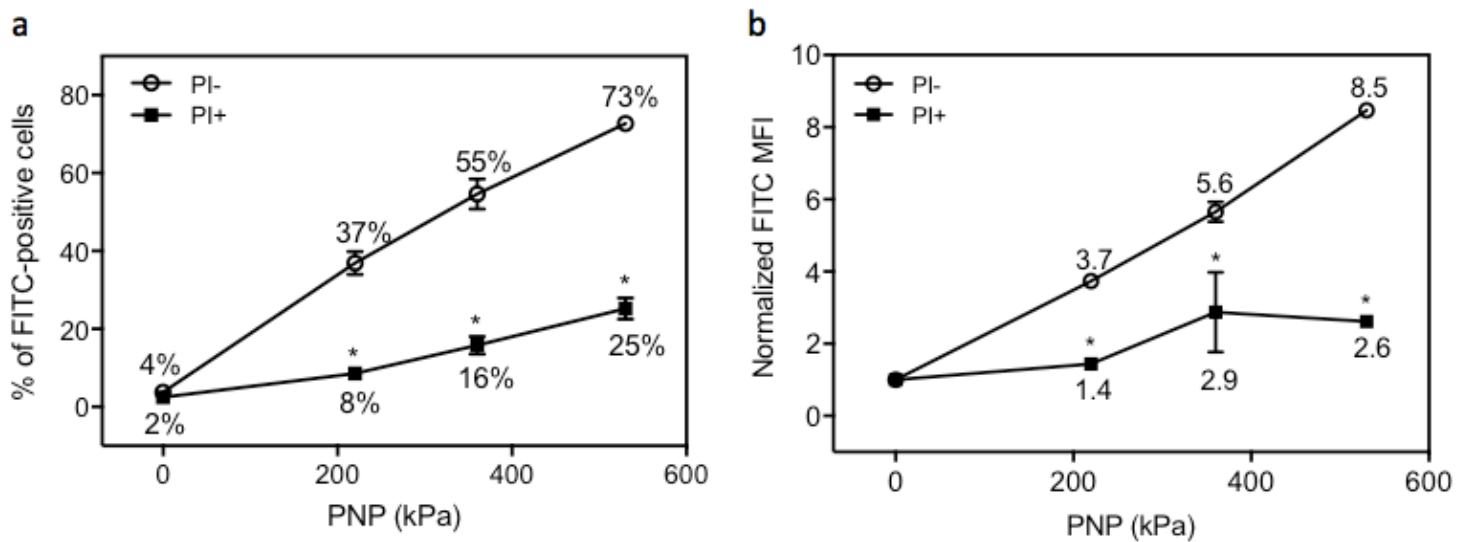

Figure 3-1: USMB-induced sonoporation with FITC-dextran. a) USMB treatment significantly increased the percentage of FITC-positive cells in populations that were (un)stained with PI. b) USMB enhanced the intracellular concentration of FITC in reversibly permeabilized cells (PI-negative). Mean values \pm SEM are shown from $n=3$ independent experiments.

\subsection{Gemcitabine dose response}

To determine BxPC3's sensitivity to gemcitabine, cells were treated with gemcitabine for 2 hours at varying concentrations (10 $\mathrm{nM}-1 \mathrm{mM})$ (Figure 3-2). Increasing chemotherapy dose increased cell cytotoxicity to a maximal cell death of $75 \%$ for doses greater than or equal to $1 \mathrm{mM}$. The interpolated IC50 dose $(1 \mu \mathrm{M})$ was used for further USMB experiments, as it resulted in a moderate percentage of cell death lower than the maximal drug response. This ensured that we were not treating cells at a dose that saturated the cellular response, so that potential USMB-mediated chemotherapy uptake could allow for further cytotoxic effects. This same dose was used for FITCdextran and 2-Chloroadenosine experiments. 


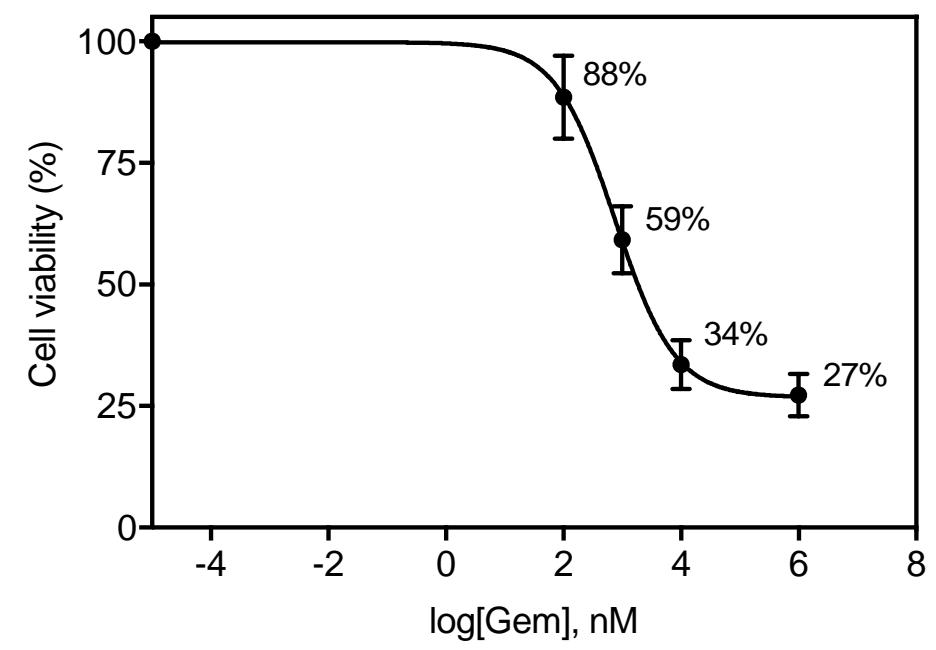

Figure 3-2: Dose-response of BxPC3 cells treated with increasing concentrations of gemcitabine. Mean cell viability percentages \pm SEM are shown from $n=3$ independent experiments.

\subsection{USMB and gemcitabine-mediated cell death}

Cell viability following treatment with $1 \mu \mathrm{M}$ of gemcitabine, USMB, and their combination is shown in Figure 3-3a. Cell viability decreased with increasing ultrasound PNP in both the presence and absence of gemcitabine. USMB in combination with gemcitabine always reduced cell viability by approximately one half the value obtained in the absence of gemcitabine $(* P<0.0001)$. Furthermore, there was no significant difference in the viability ratio (normalized with respect to their corresponding $0 \mathrm{kPa}$ condition) for cells treated in the presence and absence of gemcitabine $(P=0.98$ for comparisons made \pm GEM for each PNP), confirming that the combination therapy additively enhanced cell death (Figure 3-3b). It was expected that a higher intracellular chemotherapy concentration mediated by USMB would synergistically enhance cell death. However, the data suggest that USMB did not enhance (nor impair) the delivery of gemcitabine into BxPC3 cells under these experimental conditions. The most 
parsimonious explanation is that USMB and gemcitabine mediate cell death independently of one another.

a

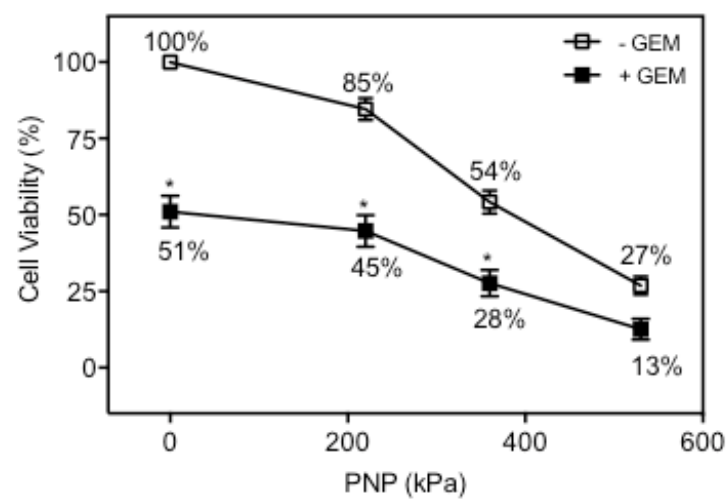

b

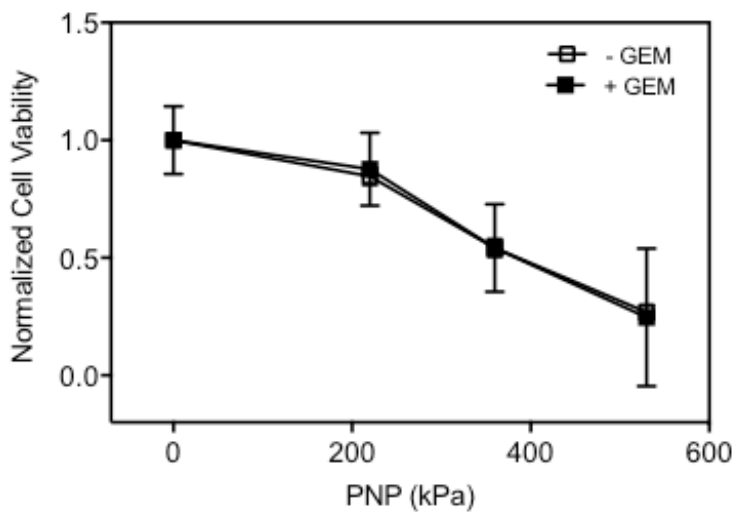

Figure 3-3: Cell viability of the combination therapy. (a) An increase in peak negative pressure ( $x$-axis) resulted in a decrease in viability ( $y$-axis) when cells were treated with USMB in the presence (closed squares) and absence (open squares) of gemcitabine. Treatment in the presence of gemcitabine always reduced cell viability relative to USMB treatment alone. (b) There is negligible difference between cell viability ratios ( $y$-axis) for cells treated in the presence and absence of gemcitabine. The above graphs show mean percentage and normalized cell viability values \pm SEM from $n=3$ independent experiments.

\subsection{USMB-induced NA uptake}

In order to verify that USMB did not enhance (nor impair) the delivery of NAs into cells, BxPC3 cells were treated with USMB in combination with $1 \mu \mathrm{M}$ of the radiolabeled nucleoside analog 2-Chloroadenosine. Radioactive accumulation due to transporter and USMB-mediated uptake was quantified by scintillation counting. The results summarized in Figure 3-4 show that there was a negligible change in the concentration of 2-Chloroadenosine within cells treated with USMB relative to unsonicated controls for both PI-negative and PI-positive populations $(P=0.6147$ and $P=0.058$, respectively). This contrasts with the expected increase in gemcitabine 
delivery given the enhanced intracellular delivery observed with an equivalent dose of FITC-dextran (Figure 3-1b). Cells that were irreversibly permeabilized retained less NA than the cells that survived USMB treatment $(* P<0.0001)$, which was consistent with the FITC-dextran analyses.

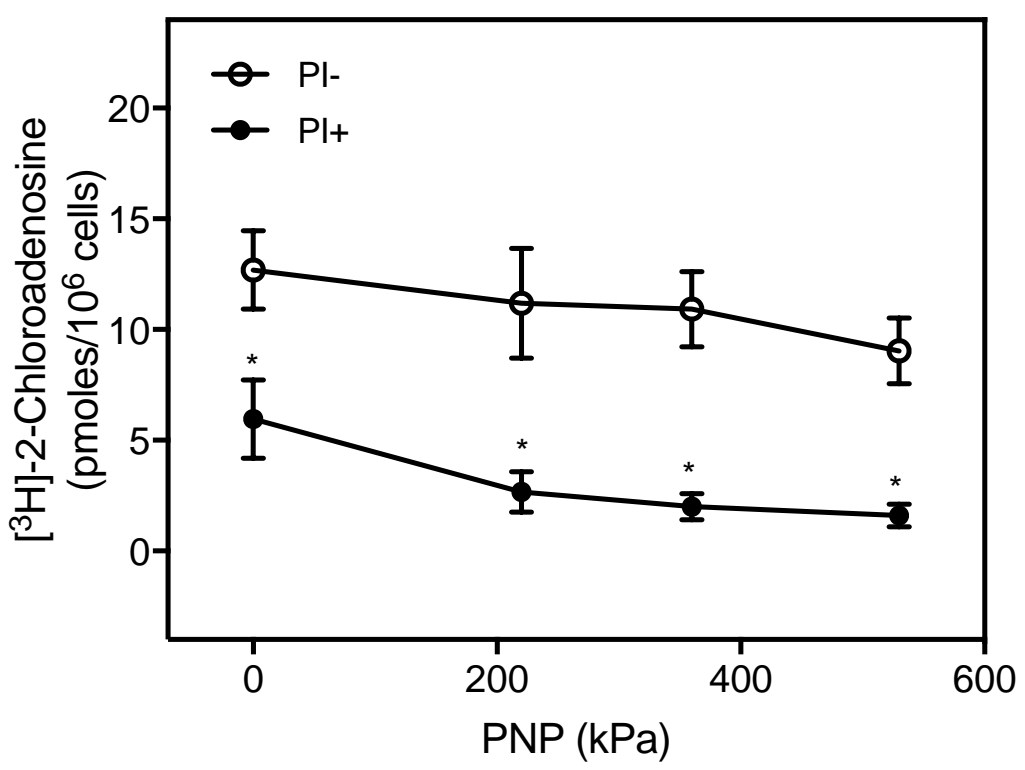

Figure 3-4: Intracellular 2-Chloroadenosine uptake. USMB therapy did not significantly alter the intracellular concentration of radiolabeled 2-Chloroadenosine in BXPC3 cells (ir)reversibly permeabilized relative to unsonicated controls. Mean intracellular 2-Chloroadenosine concentrations are shown \pm SEM from $n=3$ independent experiments.

\subsection{Acoustic response of MBs}

A passive cavitation detection system was used to monitor acoustic emissions from oscillating MBs. Figure 3-5 (a-c) shows three representative frequency spectra acquired during the 1-minute treatment for each PNP. As the PNP increased, the amplitude of the fundamental $(0.5 \mathrm{MHz})$, second $(1.0 \mathrm{MHz})$, and third $(1.5 \mathrm{MHz})$ harmonic peaks increased as well, which is indicative of increased MB non-linear 
acoustic response. Apparent broadband emission, indicated by a wide band rather than a sharp peak in the frequency spectra (i.e. an increase in amplitude in the spaces between harmonics), can be seen at the third harmonic $(1.5 \mathrm{MHz})$ for some of the frequency spectra acquired for 360 and $530 \mathrm{kPa}$. Broadband emission was not seen in the spectra obtained at $220 \mathrm{kPa}$.

The sum of the wideband area under the curve (AUC) was taken for each of the 12,000 spectra and used to quantify acoustic emission of the MBs. This wideband frequency window included harmonic and potential ultra-harmonic signals that MBs could have emitted that were within the bandwidth of the receiving transducer. Wideband acoustic emission as a function of increasing PNP is plotted in Figure 3-6. Increasing PNP correlated with the MB acoustic emission signal $\left(\mathrm{R}^{2}=0.74\right)$, which was attributed to both an increase in harmonic signal amplitudes and the presence of broadband signals at higher PNPs. 

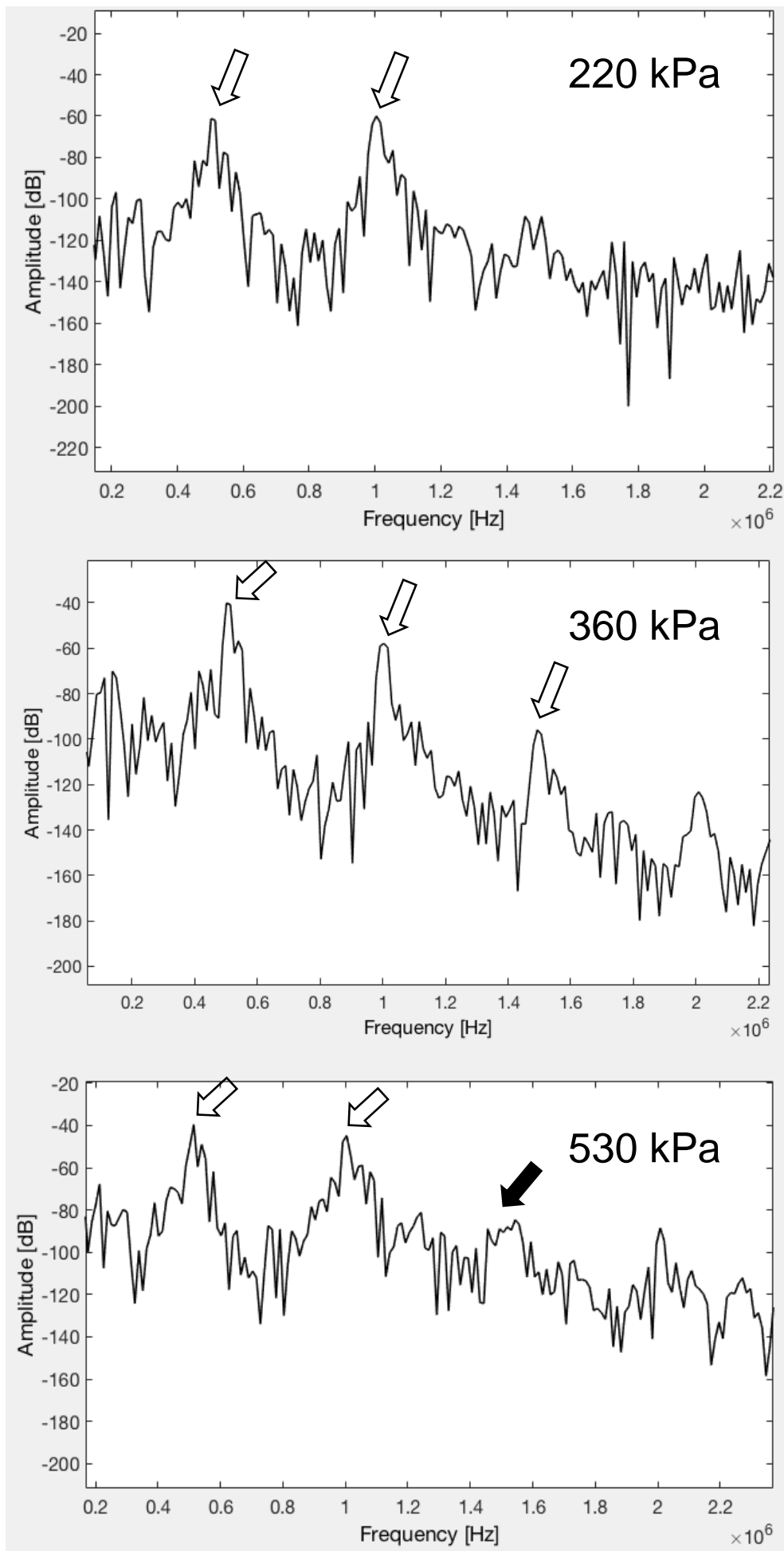

Figure 3-5: Representative frequency spectra for each PNP. The open arrows indicate the positions of fundamental and harmonic frequencies. The filled arrow indicates the location of broadband emission. 


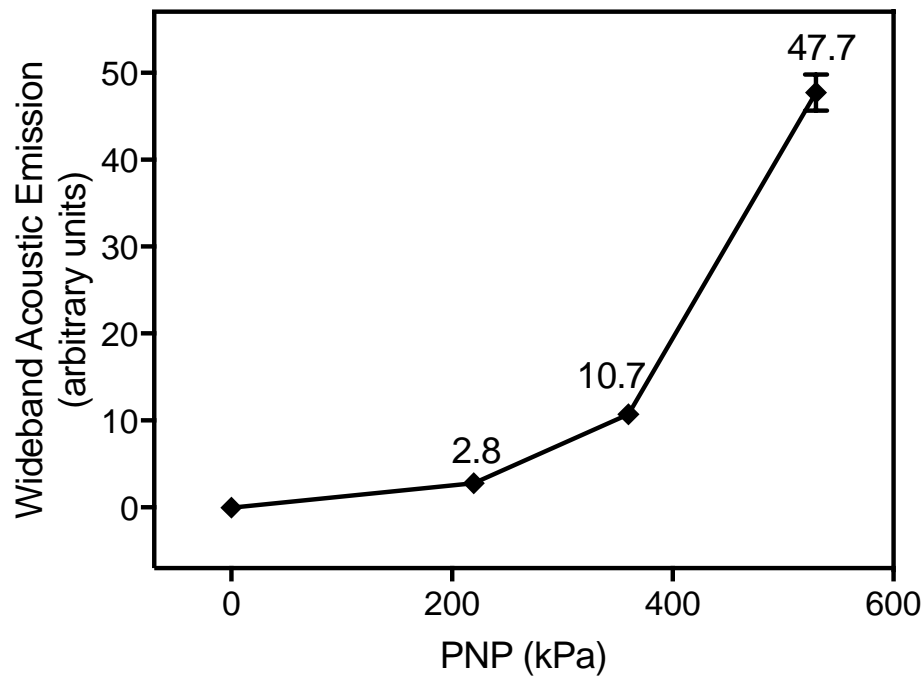

Figure 3-6: Wideband acoustic emission as a function of increasing PNP. Mean acoustic emission values are shown \pm SEM from $n=3$ independent experiments.

\subsubsection{Acoustic emission correlations with USMB-induced bioeffects}

The graphs in Figure 3-7 illustrate how MB acoustic emissions correlate with changes in USMB-induced cellular bioeffects. Table 3-1 lists the corresponding $\mathrm{R}^{2}$ values quantifying the strength of these correlations. Bearing in mind that these $\mathrm{R}^{2}$ values were calculated from only four data points, it appears that MB acoustic emissions can explain greater than $60 \%$ of the variation in the bioeffects measured - excluding intracellular concentrations of FITC and 2-Chloroadenosine in the necrotic (PI-positive) cell populations. It was expected that these necrotic populations would release their cellular constituents, which may explain this variable correlation.

An increase in the number of FITC-permeabilized cells and their intracellular concentration positively correlated with increasing MB acoustic emission (Figure 3-7a). Although few data points were used to model this trend, the data suggest that increasing MB activity can potentially be used as an indicator of sonoporation efficacy with a small 
fluorescent molecule. There was also a positive correlation between MB acoustic emissions and the percentage of dead cells (Figure 3-7c), which was anticipated given that increasing PNP positively correlated with enhanced cell death (Figure 3-3).
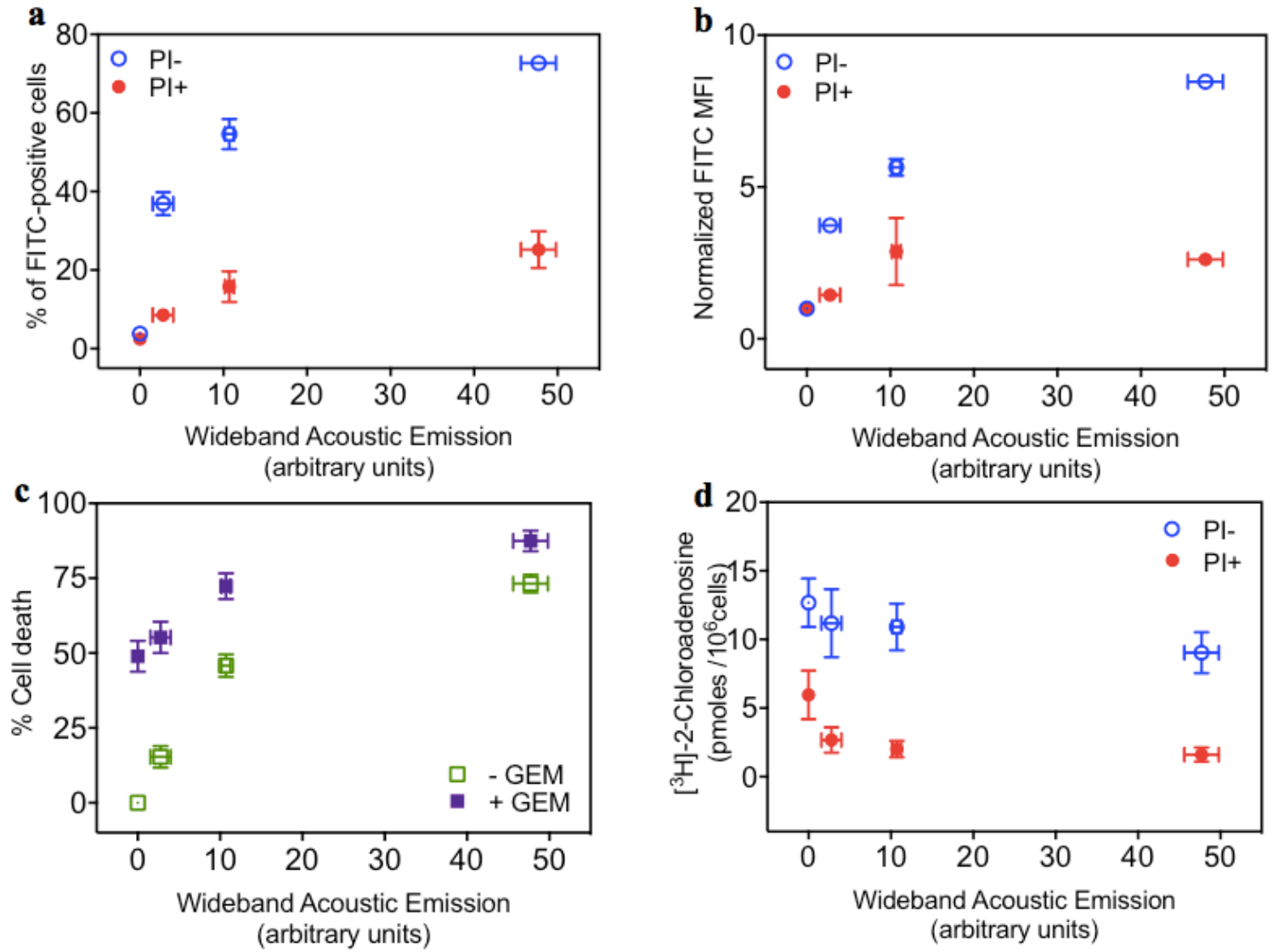

Figure 3-7: Microbubble acoustic emissions correlated with percentage of (a) FITCpermeabilized cells, (b) intracellular FITC concentration, (c) Cell Death, and (d) intracellular 2-Chloroadenosine concentration.

Table 3-1: $\mathrm{R}^{2}$ values for correlations made between MB acoustic emissions and USMBinduced bioeffects

$$
\begin{array}{cccc}
\% \text { Cell death } & \begin{array}{c}
\% \text { FITC- } \\
\text { positive cells }
\end{array} & \begin{array}{c}
\text { Intracellular } \\
\text { FITC MFI }
\end{array} & \begin{array}{c}
\text { Intracellular 2- } \\
\text { Chloroadenosine }
\end{array}
\end{array}
$$
concentration

\begin{tabular}{lllll}
\hline \hline PI - & - & 0.66 & 0.80 & 0.85 \\
\cline { 2 - 5 } PI + & - & 0.85 & 0.41 & 0.39 \\
- GEM & 0.83 & - & - & - \\
\cline { 2 - 5 } + GEM & 0.85 & - & - & -
\end{tabular}




\section{DISCUSSION}

The results from this study support the contention that ultrasonically stimulated microbubbles do not enhance the uptake (and thus cytotoxic effects) of nucleoside analogs. Given the success USMB therapy has had in enhancing the localized delivery of chemotherapy drugs ${ }^{1-3}$, it was expected that this treatment would offer the same benefits for NAs. However, this data revealed that successful sonoporation is heterogeneous and dependent on the nature of the molecule intended to be delivered.

As aforementioned, the only other studies that have employed the use of USMB with gemcitabine have shown a modest improvement in overall survival in mice and patients with pancreatic tumours ${ }^{16,18}$. Additionally, neither verified that the clinical benefits were attributed to an enhancement in gemcitabine delivery as a result of sonoporation. To address this gap in the literature, we first showed that we could successfully induce sonoporation of cancer cells in vitro using a small $10 \mathrm{kDa}(>4 \mathrm{~nm})$ FITC-dextran. Consistent with previous work, the number of permeabilized cells as well as their mean intracellular concentration (MFI) of FITC-dextran increased with increasing $\mathrm{PNP}^{6,9,56,57}$. This increase in permeabilization as a function of ultrasound pressure was due to the corresponding enhancement in MB non-linear behaviour (indicated by the increase in harmonic signal amplitudes) ${ }^{50}$, where increases in MB nonlinearity is known to correlate with increased sonoporation efficacy ${ }^{6,58}$. As the PNP increased to $530 \mathrm{kPa}$, apparent broadband emissions became evident. These were indicative of inertially cavitating $\mathrm{MBs}^{50,59,60}$, whose violent behaviour causes $\mathrm{MBs}$ to effectively puncture cells membranes, which is thought to be necessary for maximal sonoporation efficacy ${ }^{6,9,61}$. This is supported by the data which shows a maximum 
percentage of cells reversibly permeabilized (73\%) by USMB at $530 \mathrm{kPa}$, and on average, these cells retained 8.5 times more FITC-dextran relative to unsonicated samples. This increased sonoporation efficacy with increasing MB non-linearity occurred at the expense of increasing percentage of cell death ${ }^{6,9,62}$ (Figure 3-3).

It is thought that the formation of pores on cell plasma membranes induced by USMB acts as a size-exclusion barrier, only permitting entry of molecules smaller than its size ${ }^{5,63}$. And so, in theory, molecules smaller than these pores (which can be as large as $100 \mathrm{~nm}$ in diameter ${ }^{64}$ ) should enter cells more readily than molecules that are comparable and larger in size to these sieves. For instance, Karshafian et al. have shown that a larger number of cells were permeabilized by smaller FITC-dextran molecules (10 $\mathrm{kDa})$ than larger dextrans $(2 \mathrm{MDa})^{5,57}$. While investigating the effect of USMB on endocytosis, Meijering et al. also confirmed that cells internalize a greater concentration of smaller-sized fluorescent molecules $(<155 \mathrm{kDa})$ than larger ones, due to their tendency to directly diffuse through the pores ${ }^{41}$. Thus, from a geometric point of view, NAs such as gemcitabine (299.66 Da) and 2-Chloroadenosine (301.69 Da) that approximate to be less than $1 \mathrm{~nm}$ in diameter should more readily be taken up into sonicated cells than the $10 \mathrm{kDa}$ (> $4 \mathrm{~nm}$ diameter) fluorescent dextran used in this study. Additionally, one would have expected that the sonoporation efficacy of NAs should have correlated with MB behaviour as a function of increasing PNP, as it did with FITC-permeabilized cells. In contrast, this data indicated that there was no enhancement in the intracellular 2Chloroadenosine concentrations for populations (ir)reversibly permeabilized, irrespective of the ultrasound pressure. This was confirmed when we saw an additive enhancement in cell death when cells were treated with USMB and free gemcitabine. These data suggest 
that the laws of diffusion forcing small molecules across a barrier cannot solely describe the efficacy of sonoporation; there are important biological factors specific to the molecule intended to be delivered to consider as well.

This lack of enhanced NA retention in cells following USMB treatment can be explained with an understanding of gemcitabine pharmacokinetics. It is possible that the rapid flux of nucleosides into BxPC3 cells during USMB therapy can saturate the activity of the metabolic enzymes (such as $\mathrm{dCK}^{65,66}$ ) required to successfully phosphorylate gemcitabine into functional gemcitabine triphosphate (dFdCTP), where increased levels of dFdCTP are indicative of efficient metabolism to the active metabolite ${ }^{67}$. In 1991, Abbruzzese et al. alluded to the saturation of dFdCTP production as a result of high dose rates being used to treat patients in a phase-I clinical study ${ }^{39}$. Over a decade later, this concept was revisited by Tempero et al. where they were the first of many studies to show that lower doses of gemcitabine infused for longer durations led to increased production of dFdCTP in patients' peripheral blood mononuclear cells, which correspondingly lead to an increase in patients' overall survival (extended by 3 months) ${ }^{67}$. Albeit dFdCTP levels in peripheral blood mononuclear cells were not directly indicative of increased production of $\mathrm{dFdCTP}$ in tumours, but these studies demonstrated the possibility of saturating the production of active dFdCTP when using high dose rates, which likely describes what is happening when the diffusive force from USMB-induced poration is pushing free gemcitabine from the extracellular environment into cells. This confirms that just getting into cells is not enough!

The assertion that dFdCTP levels are saturated during USMB exposure, resulting in the lack of enhanced NA retention and cytotoxicity to cells in these experiments, is 
supported by the success scientists have had in overcoming gemcitabine resistance by conjugating gemcitabine onto various nanocarriers. In doing so, the mechanism of delivery into cells is no longer dependent on the presence of functional NTs at the plasma membrane and gemcitabine is now protected from inactivating enzymes such as CDA in the plasma, prolonging its longevity in the extracellular environment ${ }^{68}$. More relevantly, gemcitabine nanocarriers can increase the intracellular production of $\mathrm{dFdCTP}$ and cellular toxicity. This is believed to be due to the slower, gradual release of gemcitabine into the cell from these vectors, such that the activity of the rate limiting metabolic enzyme, $\mathrm{dCK}$, is not saturated ${ }^{68-70}$. Thus, the cytotoxic effects of gemcitabine are highly dependent on the frequency of administration, rather than enhancement in its total dosage, which is a viable way to overcome the pharmacokinetic limitations of gemcitabine chemotherapy and is worth considering for future studies using USMB with gemcitabine ${ }^{68}$.

\section{Limitations \& Future Work}

An obvious limitation of this study is that the experimental model is not a direct representation of the bioeffects that USMB and gemcitabine would induce in situ. These experiments evaluate cell viability and nucleoside uptake when USMB is applied directly to cancer cells. In actuality, sonoporation enhances the permeability of the vasculature to chemotherapy, which would permit an enhanced release of gemcitabine to the interstitial space of the tumour. Despite these findings, this can potentially explain the recent success by Kotopoulis ${ }^{71}$, employing sonoporation with gemcitabine to treat pancreatic tumours in the clinic, where the concentration of gemcitabine available to the tumour is increasing as a result of USMB. Nonetheless, this work still supports that there is a 
threshold of gemcitabine flux, beyond which, any increase in gemcitabine dose due to USMB (or other mechanisms) will not further enhance the cytotoxic effects to cells.

Another important limitation of this study concerns the fact that two different nucleoside analogs were used to measure drug cytotoxicity (gemcitabine) and intracellular accumulation (2-Chloroadenosine). Radiolabeled 2-Chloroadenosine was chosen because it is a known substrate for the physiologically relevant nucleoside transporter hENT1. However, 2-Chloroadenosine itself is not a substrate for dCK like gemcitabine is, rather it is a substrate for adenosine kinase. 2-Chloroadenosine and gemcitabine have comparable affinities for their phosphorylating enzymes $\left(\sim 1 \mathrm{uM}^{34,72}\right)$, suggesting that both may be able to saturate these enzymes to a comparable degree. However, further experiments investigating NA accumulation need to be done with radiolabeled gemcitabine to verify that there is actually no enhancement in the retention of this chemotherapy drug post-USMB treatment.

Future experiments should also be done to verify the hypothesis that USMBmediated NA uptake overwhelms the metabolic enzymes required to produce functional $\mathrm{dFdCTP}$, resulting in no intracellular change in NA concentration. To determine if this is in fact true, gemcitabine transport should be inhibited in BxPC3 cells with micromolar concentrations of nitrobenzylthioinosine ${ }^{73}$, a hENT1/2-specific inhibitor, in a sodium-free environment (to inhibit hCNT-mediated NA transport). In doing so, USMB can now be used as the sole route of gemcitabine entry into cells, without prior NT-mediated transport to saturate the enzymatic activity of dCK. It is expected that we would then see an enhancement in USMB-mediated cytotoxicity and uptake of gemcitabine. 


\section{CONCLUSION}

In conclusion, I have shown that USMB does not enhance (nor impair) the intracellular accumulation of the nucleoside analog 2-Chloroadenosine. When USMB was used to treat BxPC3 cells in vitro with the nucleoside analog gemcitabine, we saw an additive enhancement in cell death, irrespective of the ultrasound peak negative pressure. This data suggests that the two therapies mediate cell death independently of one another, where they do not synergize nor antagonize the others' mechanism of inducing cell death. 


\section{BIBLIOGRAPHY}

1. Lentacker, I., Geers, B., Demeester, J., De Smedt, S. C. \& Sanders, N. N. Design and evaluation of doxorubicin-containing microbubbles for ultrasound-triggered doxorubicin delivery: cytotoxicity and mechanisms involved. Mol. Ther. 18, 101108 (2010).

2. Canadian researchers break blood-brain barrier with new ultrasound treatment. (2016). at <http://www.ctvnews.ca/health/canadian-researchers-break-blood-brainbarrier-with-new-ultrasound-treatment-1.2648878>

3. Lee, N. G. et al. Sonoporation Enhances Chemotherapeutic Efficacy in Retinoblastoma Cells In Vitro. Investig. Ophthamology Vis. Sci. 52, 3868-3873 (2010).

4. Paproski, R. J. et al. Human Concentrative Nucleoside Transporter 3 Transfection with Ultrasound and Microbubbles in Nucleoside Transport Deficient HEK293 Cells Greatly Increases Gemcitabine Uptake. PLoS One 8, (2013).

5. Lentacker, I., De Cock, I., Deckers, R., De Smedt, S. C. \& Moonen, C. T. W. Understanding ultrasound induced sonoporation: Definitions and underlying mechanisms. Adv. Drug Deliv. Rev. 72, 49-64 (2014).

6. Bao, S., Thrall, B. D. \& Miller, D. L. Transfection of a reporter plasmid into cultured cells by sonoporation in vitro. Ultrasound Med. Biol. 23, 953-959 (1997).

7. Delalande, A., Kotopoulis, S., Postema, M., Midoux, P. \& Pichon, C. Sonoporation: Mechanistic insights and ongoing challenges for gene transfer. Gene 525, 191-199 (2013).

8. Miller, D., Bao, S., Gies, R. \& Thrall, B. Ultrasonic enhancement of gene transfection in murine melanoma tumors. Ultrasound Med. Biol. 25, 1425-1430 (1999).

9. Karshafian, R., Bevan, P. D., Williams, R., Samac, S. \& Burns, P. N. Sonoporation by Ultrasound-Activated Microbubble Contrast Agents: Effect of Acoustic Exposure Parameters on Cell Membrane Permeability and Cell Viability. Ultrasound Med. Biol. 35, 847-860 (2009).

10. Greis, C. \& Dietrich, C. F. Ultrasound Contrast Agents and Contrast-Enhanced Ultrasonography. (2016). at <http://radiologykey.com/ultrasound-contrast-agentsand-contrast-enhanced-ultrasonography/>

11. Chen, H. \& Hwang, J. Ultrasound-targeted microbubble destruction for chemotherapeutic drug delivery to solid tumors. J. Ther. Ultrasound 1, 10 (2013).

12. Kiessling, F., Fokong, S., Koczera, P., Lederle, W. \& Lammers, T. Ultrasound Microbubbles for Molecular Diagnosis, Therapy, and Theranostics. J. Nucl. Med. 53, 345-348 (2012).

13. Karshafian, R. \& Almasri, F. Enhancing chemotherapy by ultrasound and microbubbles: Effect of acoustic pressure and treatment order in in vitro suspension of reast and prostate cancer cells. ... Symp. (IUS), 2010 ... 1582-1585 
(2010). at <http://ieeexplore.ieee.org/xpls/abs_all.jsp?arnumber=5935914>

14. Lammertink, B., Deckers, R., Storm, G., Moonen, C. \& Bos, C. Duration of ultrasound-mediated enhanced plasma membrane permeability. Int. J. Pharm. 482, 92-98 (2014).

15. Watanabe, Y. et al. Low-intensity ultrasound and microbubbles enhance the antitumor effect of cisplatin. Cancer Sci. 99, 2525-2531 (2008).

16. Kotopoulis, S. et al. Sonoporation-Enhanced Chemotherapy Significantly Reduces Primary Tumour Burden in an Orthotopic Pancreatic Cancer Xenograft. Mol. Imaging Biol. (2013). doi:10.1007/s11307-013-0672-5

17. Lee, E. S. et al. Pulsed high-intensity focused ultrasound enhances apoptosis of pancreatic cancer xenograft with gemcitabine. Ultrasound Med. Biol. 39, 19912000 (2013).

18. Kotopoulis, S., Dimcevski, G., Gilja, O. H., Hoem, D. \& Postema, M. Treatment of human pancreatic cancer using combined ultrasound, microbubbles, and gemcitabine: a clinical case study. Med. Phys. 40, 072902 (2013).

19. Lee, J. Y. et al. Concurrent chemotherapy and pulsed high-intensity focused ultrasound therapy for the treatment of unresectable pancreatic cancer: initial experiences. Korean J. Radiol. 12, 176-186 (2011).

20. de Sousa Cavalcante, L. \& Monteiro, G. Gemcitabine: metabolism and molecular mechanisms of action, sensitivity and chemoresistance in pancreatic cancer. Eur. $J$. Pharmacol. 741, 8-16 (2014).

21. Kong, W., Engel, K. \& Wang, J. Mammalian nucleoside transporters. Curr. Drug Metab. 5, 63-84 (2004).

22. Young, J. D., Yao, S. Y. M., Baldwin, J. M., Cass, C. E. \& Baldwin, S. a. The human concentrative and equilibrative nucleoside transporter families, SLC28 and SLC29. Mol. Aspects Med. 34, 529-47 (2013).

23. Gray, J. H., Owen, R. P. \& Giacomini, K. M. The concentrative nucleoside transporter family, SLC28. Pflugers Arch. 447, 728-34 (2004).

24. Lostao, M. P. et al. Electrogenic uptake of nucleosides and nucleoside-derived drugs by the human nucleoside transporter 1 (hCNT1) expressed in Xenopus laevis oocytes. FEBS Lett. 481, 137-40 (2000).

25. Baldwin, S. a et al. The equilibrative nucleoside transporter family, SLC29. Pflugers Arch. 447, 735-43 (2004).

26. Baldwin, S. a et al. Functional characterization of novel human and mouse equilibrative nucleoside transporters (hENT3 and mENT3) located in intracellular membranes. J. Biol. Chem. 280, 15880-7 (2005).

27. Barnes, K. et al. Distribution and functional characterization of equilibrative nucleoside transporter-4, a novel cardiac adenosine transporter activated at acidic pH. Circ. Res. 99, 510-9 (2006).

28. DeGorter, M. K., Xia, C. Q., Yang, J. J. \& Kim, R. B. Drug transporters in drug efficacy and toxicity. Annu. Rev. Pharmacol. Toxicol. 52, 249-73 (2012). 
29. Molina-Arcas, M. \& Pastor-Anglada, M. Role of nucleoside transporters in nucleoside-derived drug sensitivity. Nucleosides. Nucleotides Nucleic Acids 29, 335-46 (2010).

30. Achiwa, H. et al. Determinants of sensitivity and resistance to gemcitabine: the roles of human equilibrative nucleoside transporter 1 and deoxycytidine kinase in non-small cell lung cancer. Cancer Sci. 95, $753-7$ (2004).

31. Mackey, J. R. et al. Functional Nucleoside Transporters Are Required for Gemcitabine Influx and Manifestation of Toxicity in Cancer Cell Lines. Am. Assoc. Cancer Res. 4349-4357 (1998).

32. Spratlin, J. et al. The Absence of Human Equilibrative Nucleoside Transporter 1 Is Associated with Reduced Survival in Patients With Gemcitabine-Treated Pancreas Adenocarcinoma The Absence of Human Equilibrative Nucleoside Transporter 1 Is Associated with Reduced Survival in. 6956-6961 (2004).

33. Santini, D. et al. Human equilibrative nucleoside transporter 1 (hENT1) levels predict response to gemcitabine in patients with biliary tract cancer (BTC). Curr. Cancer Drug Targets 11, 123-9 (2011).

34. Mini, E., Nobili, S., Caciagli, B., Landini, I. \& Mazzei, T. Cellular pharmacology of gemcitabine. Ann. Oncol. 17 Suppl 5, v7-12 (2006).

35. Huang, P., Chubb, S., Hertel, L. W., Grindey, G. B. \& Plunkett, W. Action of 2 ' , 2 ' -Difluorodeoxycytidine on DNA Synthesis. 6110-6117 (1991).

36. PharmGKB. Gemcitabine, Pharmacokinetics/Pharmacodynamics Pathway. (2003). at <https://www.pharmgkb.org/pathway/PA2036>

37. Veltkamp, S. a, Beijnen, J. H. \& Schellens, J. H. M. Prolonged versus standard gemcitabine infusion: translation of molecular pharmacology to new treatment strategy. Oncologist 13, 261-276 (2008).

38. Oberstein, P. E. \& Olive, K. P. Pancreatic cancer: why is it so hard to treat? Therap. Adv. Gastroenterol. 6, 321-337 (2013).

39. Abbruzzese, J. L. et al. A Phase-I Clinical, Plasma, and Cellular Pharmacology Study of Gemcitabine. J. Clin. Oncol. 9, 491-498 (1991).

40. Joo, W. D., Visintin, I. \& Mor, G. Targeted cancer therapy - Are the days of systemic chemotherapy numbered? Maturitas 76, 308-314 (2013).

41. Leighton, T. G. What is ultrasound? Prog. Biophys. Mol. Biol. 93, $3-83$ (2007).

42. Sirsi, S. \& Borden, M. Microbubble Compositions, Properties and Biomedical Applications. Bubble Sci Eng Technol. 1, 3-17 (2010).

43. Meijering, B. D. M. et al. Ultrasound and microbubble-targeted delivery of macromolecules is regulated by induction of endocytosis and pore formation. Circ. Res. 104, 679-87 (2009).

44. Forbes, M. M. THE ROLE OF ULTRASOUND CONTRAST AGENTS IN PRODUCING SONOPORATION. (University of Illinois at Urbana-Champaign, 2009).

45. Lindner, J. R. Microbubbles in medical imaging: current applications and future 
directions. Nat. Rev. Drug Discov. 3, 527-532 (2004).

46. Cheeke, J. D. N. Fundamentals and Applications of Ultrasonic Waves. (Taylor \& Francis Group, 2012).

47. Karshafian, R. On the Permeabilisation and Disruption of Cell Membranes by Ultrasound and Microbubbles. (University of Toronto, 2010).

48. Chen, W.-S., Matula, T. J., Brayman, A. a. \& Crum, L. a. A comparison of the fragmentation thresholds and inertial cavitation doses of different ultrasound contrast agents. J. Acoust. Soc. Am. 113, 643 (2003).

49. Berry, J. D., Burns, P. N. \& Sidhu, P. S. Microbubble Ultrasound Contrast Agents. (2016). at <http://radiologykey.com/microbubble-ultrasound-contrast-agents/>

50. Mcdannold, N., Vykhodtseva, N. \& Hynynen, K. Targeted disruption of the blood - brain barrier with focused ultrasound: association with cavitation activity. 51, 793-807 (2006).

51. Miller, D. L. et al. Overview of therapeutic ultrasound applications and safety considerations. J. Ultrasound Med. 31, 623-34 (2012).

52. Escoffre, J. M., Piron, J., Novell, A. \& Bouakaz, A. Doxorubicin Delivery into Tumor Cells with Ultrasound and Microbubbles. Mol. Pharm. 8, 799-806 (2011).

53. Iwanaga, K. et al. Local delivery system of cytotoxic agents to tumors by focused sonoporation. Cancer Gene Ther. 14, 354-363 (2007).

54. Mei, J. et al. Experimental Study on Targeted Methotrexate Delivery to the Rabbit Brain via Magnetic Resonance Imaging - Guided Focused Ultrasound. $J$. Ultrasound Med. 28, 871-880 (2009).

55. Zeng, H.-Q., Lu, L., Wang, F., Luo, Y. \& Lou, S.-F. Focused ultrasound-induced blood - brain barrier disruption enhances the delivery of cytarabine to the rat brain. J. Chemother. 24, 358-363 (2012).

56. Guzmán, H. R., Nguyen, D. X., Khan, S. \& Prausnitz, M. R. Ultrasound-mediated disruption of cell membranes. II. Heterogeneous effects on cells. J. Acoust. Soc. Am. 110, 597-606 (2001).

57. Karshafian, R., Samac, S., Bevan, P. D. \& Burns, P. N. Microbubble mediated sonoporation of cells in suspension: Clonogenic viability and influence of molecular size on uptake. Ultrasonics 50, 691-697 (2010).

58. Forbes, M. M., Steinberg, R. L. \& Jr, W. D. O. B. Frequency-Dependent Evaluation of the Role of Definity in Producing Sonoporation of Chinese Hamster Ovary Cells. J. Ultrasound Med. 30, 61-69 (2011).

59. Leinenga, G., Langton, C., Nisbet, R. \& Götz, J. Ultrasound treatment of neurological diseases - current and emerging applications. (2016). doi:10.1038/nrneurol.2016.13

60. Coussios, C. C. \& Roy, R. A. Applications of Acoustics and Cavitation to Noninvasive Therapy and Drug Delivery. Annu. Rev. Fluid Mech. 40, 395-420 (2008).

61. Delalande, A., Kotopoulis, S., Rovers, T., Pichon, C. \& Postema, M. Sonoporation 
at a low mechanical index. Bubble Sci Eng Technol. 3, 3-11 (2011).

62. Tlaxca, J. L. et al. Analysis of in vitro transfection by sonoporation using cationic and neutral microbubbles. Ultrasound Med. Biol. 36, 1907-1918 (2011).

63. Mehier-Humbert, S., Bettinger, T., Yan, F. \& Guy, R. H. Plasma membrane poration induced by ultrasound exposure: implication for drug delivery. J. Control. Release 104, 213-22 (2005).

64. Zhou, Y., Kumon, R. E., Cui, J. \& Deng, C. X. The Size of Sonoporation Pores on the Cell Membrane. Ultrasound Med. Biol. 35, 1756-1760 (2009).

65. Worden, F. P., Anthony J. Perissinotti, P. D. B. \& Bernard L. Marini, P. D. Cancer Pharmacology and Pharmacotherapy Review: Study Guide for Oncology Boards and MOC Exams. (Springer Publishing Company, 2016). at <https://books.google.ca/books?id=4b6mCwAAQBAJ>

66. Abbruzzese, J. L. New Applications of Gemcitabine and Future Directions in the Management of Pancreatic Cancer New Applications for Gemcitabine in Pancreatic Cancer. Cancer 95, 941-945 (2002).

67. Tempero, M. et al. Randomized phase II comparison of dose-intense gemcitabine: thirty-minute infusion and fixed dose rate infusion in patients with pancreatic adenocarcinoma. J. Clin. Oncol. 21, 3402-3408 (2003).

68. Celia, C., Cosco, D., Paolino, D. \& Fresta, M. Gemcitabine-loaded innovative nanocarriers vs GEMZAR: Biodistribution, pharmacokinetic features and in vivo antitumor activity. 1609-1629 (2011).

69. Wonganan, P. et al. Just Getting Into Cells is Not Enough: Mechanisms Underlying 4-(N)-Stearoyl Gemcitabine Solid Lipid Nanoparticle's Ability to Overcome Gemcitabine Resistance Caused by RRM1 Overexpression. J. Control. Release 169, 17-27 (2014).

70. Reddy, L. H. et al. A new nanomedicine of gemcitabine displays enhanced anticancer activity in sensitive and resistant leukemia types. J. Control. Release 124, 20-27 (2007).

71. Kotopoulis, S. Dialing up chemotherapy for pancreatic cancer with ultrasound. (2016). at <http://www.eurekalert.org/pub_releases/2016-05/asoa-duc051816.php>

72. Lin, B., Hurley, C. \& Fox, H. Regulation of Adenosine Kinase by Adenosine Analogs. Mol. Pharmacol. 34, 501-505 (1988).

73. Grane-Boladeras, N. Novel approaches to understanding hENT2 and hENT2related proteins: from novel nuclear variants to global networks. (2012). 